\title{
Biological Laboratory X-Ray Microscopy
}

MIKAEL KÖRDEL

Doctoral Thesis No. 11, 2020

KTH Royal Institute of Technology

Engineering Sciences

Department of Applied Physics

SE-100 44 Stockholm, Sweden 
TRITA-SCI-FOU 2020:11

ISBN 978-91-7873-555-6

Akademisk avhandling som med tillstånd av KTH framlägges till offentlig granskning för avläggande av teknologie doktorsexamen fredagen den 21 augusti 2020 klockan 13:00 i sal FD5, Albanova Universitetscentrum, Roslagstullsbacken 21, Stockholm.

(c) Mikael Kördel, maj 2020.

Front page: Cryo soft x-ray microscopy images of giant DNA virus infected Acanthamoeba castellanii. The infection times range from 12 hours (top left) to 72 hours (bottom right). The ovoid viral particles can be seen replicating inside the cells until they take up a large part of the cell volume. All images were acquired using the Stockholm laboratory $x$-ray microscope. (Figure adapted from Paper D). 


\section{Abstract}

Soft $\mathrm{x}$-ray microscopy in the water window $(\lambda \approx 2.3-4.3 \mathrm{~nm})$ is a powerful technique for high-resolution biological imaging. The strong natural contrast between carbon-based structures and water allows visualization of hydrated and unstained samples, while providing enough transmission through up to $\sim 10 \mu \mathrm{m}$ of organic matter. Furthermore, the full potential of this technique can be exploited by performing computed tomography, thus obtaining a complete 3D image of the object.

Routine short-exposure water-window microscopy of whole cells and tissue is currently performed at synchrotron-radiation facilities around the world, but with a limited accessibility to the wider research community. For this reason, laboratory-based systems have been developed, which are now reaching maturity. The benefits compared to the synchrotron-based instruments include easier integration with complementary methods in the home laboratory, in addition to the increased access that allows for the often time-consuming optimization of experimental parameters as well as longitudinal studies.

This Thesis presents recent developments of the Stockholm laboratory $\mathrm{x}$-ray microscope as well as several biological applications. Work has been done on improving the mechanical and thermal stability of the microscope, resulting in a resolution of $25 \mathrm{~nm}$ (half period) in images of test targets. The biological applications were enabled by a significantly increased x-ray flux through the system as well as an improved operational stability. This work demonstrates 10-second exposure imaging of whole cryofixed cells, imaging of viral infections in cells, and 20 minutes total exposure cryotomography. 


\section{Sammanfattning}

Röntgenmikroskopi i vattenfönstret $(\lambda \approx 2.3-4.3 \mathrm{~nm})$ är en kraftfull metod för högupplöst biologisk avbildning. Den naturligt höga kontrasten mellan kolbaserade strukturer och vatten möjliggör visualisering av prover i ett nästintill opåverkat tillstånd, och ger samtidigt tillräcklig transmission genom upp till $\sim 10 \mu \mathrm{m}$ organisk materia. Teknikens fulla potential utnyttjas vidare genom datortomografi, vilket resulterar i en fullständig 3D-bild av objektet.

Röntgenmikroskopi i vattenfönstret, av celler och vävnad och med kort exponeringstid, utförs rutinmässigt vid synkrotronljuskällor runt om i världen, men med begränsad tillgänglighet för forskarsamfundet. Av den anledningen har laboratoriebaserade system utvecklats, vilka nu börjar nå mognad. Fördelarna jämfört med synkrotronbaserade instrument består av enklare integrering av komplementära laboratoriemetoder, utöver den utökade tillgängligheten som tillåter tidskrävande optimering av experimentella parametrar såväl som longitudinella studier.

Denna avhandling beskriver nyligen utfört arbete för att förbättra det kompakta mjukröntgenmikroskopet i Stockholm, samt flera biologiska tillämpningar. Arbete har gjorts för att förbättra mikroskopets mekaniska och termiska stabilitet, vilket har resulterat i $25 \mathrm{~nm}$ upplösning (halv period) i bilder av teststrukturer. De biologiska tillämpningarna har möjliggjorts av en markant ökad röntgenintensitet vid provet, såväl som förbättrad driftsstabilitet. Resultaten som presenteras består, bland annat, av avbildning av kryofixerade hela celler med 10-sekundersexponeringar, avbildning av virusinfektioner i celler och kryotomografi med 20 minuters total exponeringtid. 


\section{List of papers}

\section{Paper A}

E. Fogelqvist, M. Kördel, M. Selin, and H. M. Hertz, Stability of liquidnitrogen-jet laser-plasma targets, J. Appl. Phys. 118, 174902 (2015).

\section{Paper B}

E. Fogelqvist, M. Kördel, V. Carannante, B. Önfelt, and H. M. Hertz, Laboratory cryo $x$-ray microscopy for $3 D$ cell imaging, Sci. Rep. 7, 13433 (2017).

\section{Paper C}

M. Kördel, K. G. Y. Arsana, H. M. Hertz, and U. Vogt, Stability investigation of a cryo soft $x$-ray microscope by fiber interferometry, Rev. Sci. Instrum. 91, 023701 (2020).

\section{Paper D}

M. Kördel, M. Svenda, H. K. N. Reddy, E. Fogelqvist, B. Hamawandi, M. S. Toprak, H. M. Hertz, and J. A. Sellberg, Quantitative bioconversion in giant DNA virus infection, submitted manuscript (2020).

\section{Paper E}

M. Kördel, A. Dehlinger, C. Seim, U. Vogt, E. Fogelqvist, J. A. Sellberg, H. Stiel, and H. M. Hertz, Laboratory water-window x-ray microscopy, Optica, accepted manuscript (2020).

\section{Paper F}

A. Dehlinger, C. Seim, H. Stiel, S. Twamley, A. Ludwig, M. Kördel, D. Grötztsch, S. Rehbein and B. Kanngießer, Laboratory soft x-ray microscopy with an integrated visible-light microscope - Correlative workflow for faster $3 D$ cell imaging, submitted manuscript (2020). 


\section{Other publications}

The author has contributed to the following publications, which are related to this Thesis but have not been included in it.

M. Kördel, E. Fogelqvist, V. Carannante, B. Önfelt, H. K. N. Reddy, K. Okamoto, M. Svenda, J. A. Sellberg, and H. M. Hertz, Biological Laboratory X-ray Microscopy, Microsc. Microanal. 24(S2), 346-347 (2018).

M. Kördel, E. Fogelqvist, V. Carannante, B. Önfelt, H. K. N. Reddy, M. Svenda, J. A. Sellberg, K. G. Y. Arsana, U. Vogt and H. M. Hertz, Biological laboratory $x$-ray microscopy, Proc. SPIE 11112, 111120T (2019). 


\section{Contents}

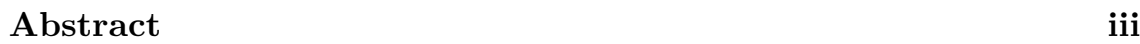

Sammanfattning iv

$\begin{array}{ll}\text { List of papers } & \text { v }\end{array}$

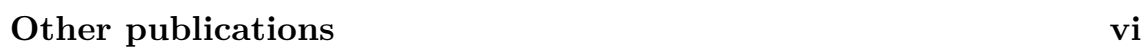

\begin{tabular}{|l|l}
\hline List of figures & ix
\end{tabular}

\begin{tabular}{lll}
\hline & Introduction & $\mathbf{1}$
\end{tabular}

1.1 Resolution, contrast and signal-to-noise . . . . . . . . . . 2

1.2 High-resolution microscopy techniques . . . . . . . . . . . . . . . . . 4 4

1.2.1 Electron microscopy . . . . . . . . . . . . . . . . . . . 4

1.2 .2 Atomic force microscopy . . . . . . . . . . . . . . . 5

1.2 .3 Super-resolution microscopy . . . . . . . . . . . . . . . 5

1.2 .4 X-ray methods . . . . . . . . . . . . . . . . . . . 6

1.3 This Thesis . . . . . . . . . . . . . . . . . . . 8

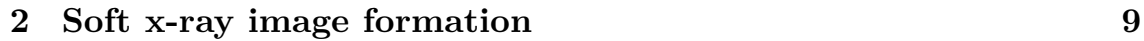

2.1 Soft x-ray interaction with matter . . . . . . . . . . . . . . 99 9

2.1 .1 The water window . . . . . . . . . . . . . . 11

2.1 .2 Soft x-ray reflectivity . . . . . . . . . . . . . . . . . . . . . . . . . . 12

2.2 Image formation in 2D . . . . . . . . . . . . . . . . . . . 13

2.2.1 $\quad$ Projection imaging . . . . . . . . . . . . . . . . . 13

2.2 .2 Imaging by a lens . . . . . . . . . . . . . . . . . . . . . . . . . . . . . . 14

2.2 .3 Coherent diffraction imaging . . . . . . . . . . . . . 18

2.3 Tomographic (3D) image formation . . . . . . . . . . . . . . . 19

2.3 .1 The Fourier slice theorem . . . . . . . . . . . . . . . . 19

2.3 .2 Tomographic reconstruction . . . . . . . . . . . . 20

2.3 .3 Resolution in tomography . . . . . . . . . . . . . 21 
3 Soft x-ray microscopy 23

3.1 Scanning or full-field . . . . . . . . . . . . . . . . . . . . 23

3.2 Soft x-ray sources . . . . . . . . . . . . . . . . . . . . 24

3.2 .1 Synchrotron sources . . . . . . . . . . . . . . 25

3.2 .2 Free-electron lasers . . . . . . . . . . . . . . . . . . . . 27

3.2 .3 Laboratory x-ray sources . . . . . . . . . . . . . . . 28

$3.3 \quad$ Soft x-ray optics $\ldots \ldots \ldots \ldots \ldots \ldots$

$3.3 .1 \quad$ Multilayer mirrors . . . . . . . . . . . . . . . . 30

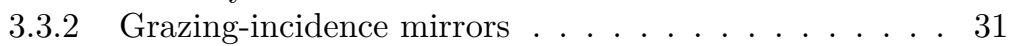

$3.3 .3 \quad$ Zone plates $\ldots \ldots \ldots \ldots$. . . . . . . . . . . . . . 33

3.4 Optical arrangements for TXM . . . . . . . . . . . . . . 36

3.5 Sample environment . . . . . . . . . . . . . . . . . . 37

$3.5 .1 \quad$ Cryofixation . . . . . . . . . . . . . . . . 38

3.5 .2 Chemical fixation . . . . . . . . . . . . . . . . . 38

3.5 .3 Wet imaging . . . . . . . . . . . . . . . . . . . . . 39

$3.6 \quad$ X-ray detection $\ldots \ldots \ldots \ldots$

\begin{tabular}{|lll}
4 & The Stockholm laboratory x-ray microscope & 41
\end{tabular}

4.1 Microscope arrangement . . . . . . . . . . . . . . . . . 41

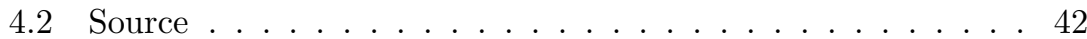

4.2 .1 Source stability . . . . . . . . . . . . . . . . . 43

4.3 Condenser mirror . . . . . . . . . . . . . . . . . . . . 44

4.4 Objective zone plate . . . . . . . . . . . . . . . . 45

4.5 Sample stage $\ldots \ldots \ldots \ldots \ldots$. . . . . . . . . . . . . . . . . . . . . . . . . .

4.6 Detector $\ldots \ldots \ldots \ldots \ldots$

4.7 Vacuum system . . . . . . . . . . . . . . . . . . . . . . . 47

4.8 System stability $\ldots \ldots \ldots \ldots \ldots$

5 Biological applications $\quad 49$

5.1 Starvation-induced autophagy in HEK 293T cells . . . . . . . 49

$5.2 \quad$ NK-cell target-cell interaction $\ldots \ldots \ldots \ldots \ldots$

5.3 Giant DNA virus infection $\ldots \ldots \ldots \ldots \ldots$. . . . . . . 52

$5.4 \quad$ Glycocalyx of THP-1 cells $\ldots \ldots \ldots \ldots \ldots$

5.5 Future applications . . . . . . . . . . . . . . . . 54

6 Conclusions and outlook $\quad 55$

\begin{tabular}{ll}
\hline Summary of papers & 57
\end{tabular}

\begin{tabular}{ll}
\hline Acknowledgements & 59
\end{tabular}

\begin{tabular}{ll}
\hline References & 61
\end{tabular} 


\section{List of figures}

1.1 The effects of resolution, contrast and SNR in an image. . . . . . . 3

1.2 Electromagnetic spectrum from $1 \mathrm{eV}$ to $10 \mathrm{keV}(\lambda=1 \AA-1 \mu \mathrm{m})$. 7

$2.1 \quad$ Atomic cross sections of carbon. . . . . . . . . . . . . . . . 10

$2.2 \quad$ X-ray attenuation in the water window. . . . . . . . . . . . . . 12

2.3 Coherent and incoherent imaging of a Siemens star. . . . . . . . 17

2.4 The Rayleigh resolution criterion. . . . . . . . . . . . . . . 18

2.5 The Fourier slice theorem. . . . . . . . . . . . . . . . . . . . 20

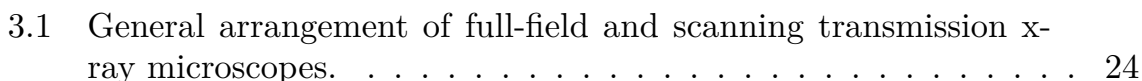

3.2 Synchrotron radiation sources. . . . . . . . . . . . . 25

3.3 Laboratory soft x-ray sources. . . . . . . . . . . . . . . . . . . . . . . . . . . 28

3.4 Reflective x-ray optics. . . . . . . . . . . . . . . . . . . 32

3.5 Fresnel zone plate working principle. . . . . . . . . . . . . . 33

$3.6 \quad$ Optical arrangements for transmission x-ray microscopy. . . . . . 37

4.1 The Stockholm laboratory x-ray microscope arrangement. . . . . 42

4.2 Setup for real-time flash-imaging of the liquid-nitrogen jet. . . . 43

4.3 Reflectivity measurement of the multilayer condenser mirror. . . 45

4.4 Images of a gold Siemens star, illustrating the improved resolution. 48

5.1 Monitored plunge-freezing setup. . . . . . . . . . . . . . . 50

5.2 Laboratory cryo x-ray microscopy of HEK $293 \mathrm{~T}$ cells. . . . . . . 51

5.3 Laboratory cryo x-ray microscopy of NK-cell HEK-cell interaction. 52

5.4 Laboratory cryo x-ray microscopy of giant DNA virus infected Acanthamoeba Castellanii. . . . . . . . . . . . . . . . . 53 



\section{Chapter 1}

\section{Introduction}

To visually observe a system or a process is often the most efficient way to learn about it. In some cases it is enough to make direct observations with the naked eye and use adequate means to document the observations. This kind of simple empirical research has led to many scientific advances, but has some obvious limitations. The challenge lies in observing what the eye cannot see. This may be an object too faint and distant or too small to be resolved by the eye, or perhaps a process too fast for us to register.

In biological research the main challenge often lies in the small size of the investigated objects, and hence, the invention of the compound microscope in the early $17^{\text {th }}$ century opened up a world of possibilities [1. Dutch spectacle makers, among them Zacharias Jansen and Hans Lipperhey, had been experimenting with lenses in tubes and discovered that certain combinations made objects appear greatly magnified. The Italian polymath Galileo Galilei heard of the attempts and in 1609 he constructed the compound microscope, consisting of a positive objective lens, a negative eyepiece and a focusing device. Antoni van Leeuwenhoek is usually accredited for establishing microscopy as a tool in biological sciences. Even though he only used a simple single-lens microscope he managed to observe, e.g., muscle fibers and red blood cells, and in 1677 he became the first to publish observations of microorganisms [2] Great contributions to microscopy and microbiology were also made by Robert Hooke, who further improved the compound microscope and used it to study small insects, plants and seeds. In his famous book Micrographia, from 1665, Hooke published detailed drawings of his observed specimen and also coined the term cell, while describing the microscopic texture of cork [3].

The construction and development of microscopes were for a long time almost exclusively based on empirical principles. This changed in the late $19^{\text {th }}$ century, when Ernst Abbe established the theoretical foundations of 
light microscopy, including diagnosis and correction of optical aberrations, i.e., deviations from an ideal image formation [4]. Furthermore, he defined the theoretical resolution limit for a (full-field) light microscope due to the diffraction of light through the objective lens. He concluded that the minimum resolvable distance $r$ depends on the wavelength of light $\lambda$ and the numerical aperture $N A$ of the objective, according to

$$
r=\frac{\lambda}{2 N A} .
$$

For an objective in air the numerical aperture is simply the sine of the collection angle, which effectively limits the achievable resolution to half the wavelength of visible light. Together with Carl Zeiss, Abbe used his theory to construct the first diffraction-limited microscope [1]. In 1900 August Köhler joined Zeiss and introduced the illumination scheme currently used in light microscopy, known as Köhler illumination [5]. At this point, the field of microscopy had reached an important milestone, and furthermore a seemingly impenetrable limitation in resolving power.

\subsection{Resolution, contrast and signal-to-noise}

Before making any detailed comparisons between modern microscopy techniques, a few basic concepts need to be defined. These will form a basis for any discussions on image quality in this Thesis.

Resolution has already been mentioned and is commonly discussed in the context of microscopy. It simply describes the ability of the imaging system to resolve small details in the imaged object. This can be limited by factors like diffraction through a lens, a too large pixel size, or if the object moves while the image is acquired. High resolution is key to making a useful microscope. However, the resolution itself provides very little information without also considering the contrast.

The contrast describes how much a feature stands out in an image, by the difference in color or light intensity, compared to the background. This Thesis considers mostly gray-scale images, and thus, the light intensity is the relevant parameter here. The contrast must originate from intrinsic properties of the observed objects, like the ability to absorb light more strongly than its surroundings. Furthermore, the ability of the imaging system to transfer the intrinsic contrast to the image will typically depend on the size of the imaged features, often described in terms of their spatial frequencies [6]. As a result, the resolution provided by an imaging system must be defined in terms of the transferred contrast.

Note that the image contrast, as well as the average brightness over the whole image, can easily be adjusted in a digital image, using any image 

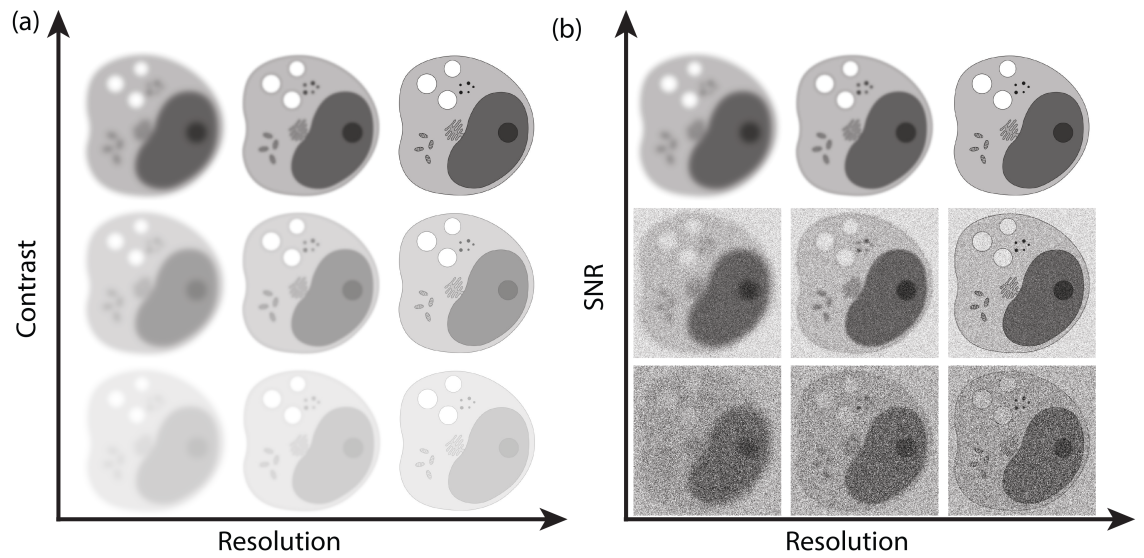

Figure 1.1. The effects of resolution, contrast and signal-to-noise ratio (SNR) in an image of a simple cell model. (a) Contrast versus resolution. (b) SNR versus resolution.

processing software. Now it might sound like any feature in a digital image can be seen with perfect contrast as long it can be resolved. There are two main reasons why this is not the case. The first reason is that increasing the contrast to observe a low-contrast feature typically results in erasing the contrast in other parts of the image, which become very dark or very bright. The second reason is more fundamental and is related to the concept of noise.

Noise is the unwanted but unavoidable random fluctuations in signal, present in any imaging system, including the human eye. It can arise at the detection step, as electronic noise, at the sample or due to a fluctuating illumination. In many imaging techniques the primary source of noise is quantum or shot noise, as a consequence of the limited number of particles (e.g., electrons or photons) that reach the detector. Regardless of origin, all contributions to the total noise will add a randomness to the image, which will be amplified with any attempts to increase the contrast after the image is acquired. Here it is appropriate to define the signal-to-noise ratio (SNR), which is the ratio between the image signal and noise amplitude. A higher SNR usually means a better and clearer image. Figure 1.1 illustrates the influence of resolution, contrast and SNR on an image of a simple cell model. 


\subsection{High-resolution microscopy techniques}

In the $20^{\text {th }}$ century, the desire to study even smaller things led to the development of alternative microscopy techniques. Fluorescent markers and advanced illumination schemes were used to improve the contrast of certain objects and enabled optical sectioning for a 3D view of thick samples. Building on these techniques, super-resolution microscopy was developed, which cleverly circumvents the diffraction limit 7 . By moving away from visible light to smaller wavelengths (e.g., x-rays) [8], or using electrons to probe the samples, even better resolution was achieved. An important feature of the modern techniques, compared to early light microscopy, is that the images have to be registered on a medium, rather than being directly observed by the eye. Today this medium is often (but not always) a highresolution camera, or detector, that captures and transfers discrete digital information about the image.

The ever-growing number of imaging techniques results in a range of contrast mechanisms, suitable for different imaging applications. It is therefore important to not only focus on the best achievable resolution when deciding on an imaging technique for a certain application.

\subsubsection{Electron microscopy}

Electrons are subatomic particles, but they also exhibit wave-like properties, like diffraction. These properties are determined by their de Broglie wavelength [9], defined by $\lambda=h / p$, where $h$ is the Planck constant and $p$ is the momentum of the particle. Already at moderate acceleration voltages the electron momentum is enough to result in a wavelength shorter than the size of an atom $(\sim 0.1 \mathrm{~nm})$, thus making electrons attractive for high-resolution imaging.

Electron microscopes use the fields from electrostatic and magnetic lenses to focus the electrons, in a manner similar to glass lenses in light microscopes. Just like the glass lenses, the electric and magnetic fields can induce aberrations, which typically limit the resolution at higher acceleration voltages 10 . In single-particle cryogenic electron microscopy (cryo-EM), however, practically aberration-free imaging can be achieved by combining the information from a large number of identical particles [11]. Technical developments in this field were recently awarded a Nobel prize. However, the applications in this Thesis mainly involve larger and more heterogeneous objects, and therefore this technique will not be further covered here.

Conventional electron microscopy (EM) is either done in scanning or transmission mode. A scanning electron microscope (SEM) scans the surface of an object, using a focused electron beam, and detects the secondary 
electrons that are scattered off the material. With a highly focused beam, the topology and composition of the object surface can be imaged with a resolution down to about $1 \mathrm{~nm}[12$. In transmission electron microscopy (TEM), the focused electrons are instead transmitted through the (thin) object and detected on the other side. The contrast is based on the absorption of electrons in the sample and the resolution can reach as far as $50 \mathrm{pm}$ for certain samples 10 .

An important characteristic of the electrons is their extremely short penetration depth in most materials. For SEM, this is beneficial since the detected secondary electrons mainly originate from the focus spot on the object surface. In TEM, however, this sets a limit to the thickness of the imaged sample. Specimen should typically be no more than a few $100 \mathrm{~nm}$ thick to allow enough transmission [13, meaning that thicker samples, like cells or tissue, have to be sectioned (sliced). This process includes complicated and tedious sample preparation procedures, including chemical or cryogenic fixation, staining and embedding in a medium.

\subsubsection{Atomic force microscopy}

Atomic force microscopy (AFM) uses a cantilever with an extremely sharp tip to probe a sample surface in a scanning manner [14]. The fine movements of the cantilever are registered by a sensitive piezoelectric device in order to map the topology of the surface. The resolution in AFM can reach below $1 \mathrm{~nm}$, but the method is intrinsically limited to surface measurements.

In addition to the surface topology AFM can be used to probe the mechanical stiffness in, e.g., biological samples like cells and tissue [15].

\subsubsection{Super-resolution microscopy}

The term super-resolution microscopy (SRM) refers to a number of techniques that enable visible-light imaging with higher resolution than the diffraction limit, as stated by Ernst Abbe. Common for all SRM techniques is that they use fluorescent staining in combination with a clever illumination or detection scheme in order to circumvent the diffraction limit. The different techniques are commonly divided into two major groups that utilize either deterministic or stochastic schemes [16].

The deterministic techniques use different kinds of structured illuminations to put the fluorescent staining molecules in on or off states, meaning that they do or do not emit light. In stimulated emission depletion (STED) microscopy [17, the fluorescent molecules in a certain spot on the sample are first excited by the excitation laser. In the next step, a depletion laser illuminates a ring around the same spot, which works to stimulate the emission in all parts but the very center of the spot. The emission from this 
central area, which can be made smaller than the diffraction-limited focus, is subsequently detected.

The stochastic SRM techniques rely on random emission from sparsely distributed fluorescent molecules, which over time gives information about their exact position. In photoactivated localization microscopy (PALM) [18], a full-field illumination activates the fluorescent molecules. A large number of images are then acquired and computationally processed to determine the position of the molecules. A similar technique, called stochastic optical reconstruction microscopy (STORM) [19 uses special buffers to chemically induce excitation, thus eliminating the need for photo-activation.

Both the deterministic and stochastic SRM techniques have demonstrated impressive resolutions down to the 20-nm range [20,21]. However, it is once again important to consider the contrast mechanism and what is actually being imaged. For the stochastic methods, the ability to separate individual dyes is not the same thing as resolving a physical feature. The resolution in a biological specimen depends on the labelling density and the switching properties of the dye, and is typically limited to at least double the distance between the just resolvable dyes [16]. Also the deterministic techniques rely heavily on the ability to label the specific structures with the appropriate densities. Furthermore, high-resolution imaging using, e.g., STED, requires high light intensities up to $1 \mathrm{GW} / \mathrm{cm}^{2}$, which can result in strong photobleaching of the fluorescent dyes and even phototoxicity [22].

Many SRM techniques also allow 3D imaging. Here, the resolution varies between the different methods and depending on the thickness of the sample 7,23 . Providing high-resolution 3D information about an object also typically means very long exposure times, especially for larger sample volumes 7 .

\subsubsection{X-ray methods}

In 1895, around the same time as Zeiss and Abbe achieved the first diffraction-limited light microscope, Wilhelm Röntgen discovered "a new kind of radiation" while experimenting with discharge tubes [24]. For the sake of brevity, and to point out the unknown nature of the radiation, Röntgen decided to use the name $x$-rays (in German $X$-Strahlen). The medical application of x-rays was immediately recognized by Röntgen and others, and already a few months after the original publication exploration of the new possibilities was initiated at hospitals and research facilities across Europe and North America 25]. Convincing evidence of the electromagnetic-wave nature of x-rays was first produced in 1912 by Max von Laue, who discovered that the x-rays could interfere to create diffraction patterns when passing through crystals $[26$. His findings would 


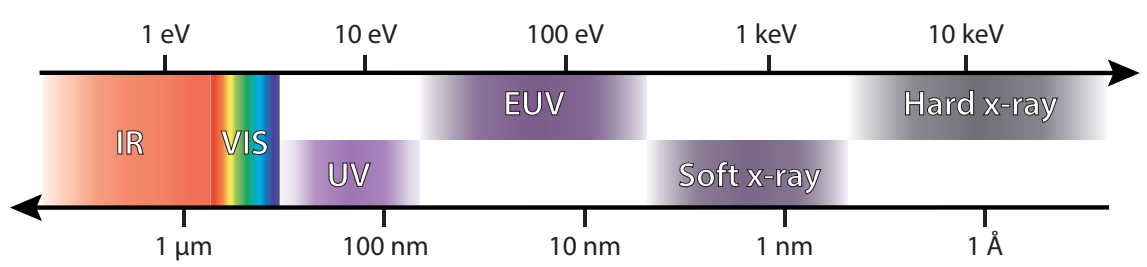

Figure 1.2. The electromagnetic spectrum from $1 \mathrm{eV}$ to $10 \mathrm{keV}(\lambda=1 \AA-1 \mu \mathrm{m})$. This energy range consists of infrared (IR), visible (VIS), ultraviolet (UV), extreme ultraviolet (EUV), soft x-ray and hard x-ray radiation.

eventually be used in the field of x-ray crystallography.

$\mathrm{X}$-rays are found in the shorter-wavelength part of the electromagnetic spectrum compared to visible light, as depicted in Fig. 1.2 Equivalently, $\mathrm{x}$-rays can be said to have higher photon energies. This is the wave-particle duality that applies to any electromagnetic radiation. Since x-rays span many orders of photon energy, their interaction with matter will vary, both in nature and degree [27, 28]. Generally, hard $x$-rays, with photon energies from several $\mathrm{keV}$ and up, will interact less and consequently penetrate deeper into objects. This is the property that originally made $\mathrm{x}$-rays attractive for medical imaging purposes. Soft $x$-rays $(250 \mathrm{eV}$ to few $\mathrm{keV})$ tend to interact more and will, thus, be absorbed over rather short distances. This property can be utilized for microscopy purposes, since it enables a high contrast in very small objects.

\section{X-ray microscopy}

X-ray microscopy (XRM) was enabled in the second half of the $20^{\text {th }}$ century by the invention of new and brighter x-ray sources and focusing $\mathrm{x}$-ray optics [8]. XRM can refer to a number of techniques, including contact x-ray microscopy 29, full-field transmission x-ray microscopy (TXM) 30. and scanning transmission x-ray microscopy (STXM) [31]. Contact x-ray microscopy is rarely used today, while TXM and STXM regularly demonstrate significant results in fields like cell biology, material science and environmental science 32,33 . The resolution achieved by these techniques currently approach the 10-nm level 34,35].

With the more recent coherent x-ray sources another branch of lensless XRM opened up [36]. Plane-wave coherent diffraction imaging (CDI) [37], x-ray holography [38, and ptychography [39] are some of the major lensless XRM techniques. 


\subsection{This Thesis}

The main focus of this Thesis is lens-based soft x-ray microscopy, in which soft x-rays and specialized x-ray optics are used to form a real image of the object. Although this technique has many similarities to visible-light microscopy in its arrangement, most of the components are fundamentally different. To understand why this is the case, knowledge about soft x-ray interaction with matter and soft x-ray image formation is required. This is outlined in Chapter 2 .

Chapter 3 proceeds with a general description of the soft x-ray microscope and its main components, from the x-ray source to optics and sample environment. The Stockholm laboratory x-ray microscope is the main instrument used for the imaging applications. Details on its setup, performance and recent improvements are given in Chapter 4. The imaging applications are mainly biological and the samples include cryofixed whole cells, viruses and virus-infected cells, and cell-cell interactions. A review of the biological applications from the last few years, including an outlook on future projects, is given in Chapter 5 


\section{Chapter 2}

\section{Soft x-ray image formation}

This chapter briefly summarizes the fundamentals of soft x-ray interaction with matter as well as the formation of images in 2D and 3D. Much of the theory is applicable to electromagnetic radiation in general, but focus will be on soft x-rays, with photon energies ranging from about $250 \mathrm{eV}$ to few $\mathrm{keV}(\lambda \approx 0.5-5 \mathrm{~nm})$. This will serve as a theoretical background for a better understanding of the soft x-ray microscope, which is described in Chapter 3

\subsection{Soft x-ray interaction with matter}

In x-ray imaging the contrast arises due to the ability of matter, or more specifically the electrons, to interact with the x-rays. When a certain object interacts to a higher (or lower) degree than its surroundings, contrast appears, and it is a matter for the imaging system to transfer that contrast to the detection step.

There are three possible ways for electromagnetic radiation to interact with the bound electrons in matter: elastic scattering, inelastic scattering and photoelectric absorption [40]. Elastic scattering means that the electron, driven by the accelerating force of the incident electric field, reemits radiation of the same frequency. By doing so, the incident radiation keeps its original wavelength, but changes its direction. Elastic scattering involving free electrons is usually called Thomson scattering, while the process involving bound electrons is referred to as Rayleigh scattering. Inelastic scattering, or Compton scattering, cannot be explained by classical electromagnetism and instead the particle nature of electromagnetic radiation is considered. In this regime an incident photon transfers a part of its original energy $E$ to a bound electron, and then continues at angle $\theta$ from its original trajectory. For this process to take place the photon must have 


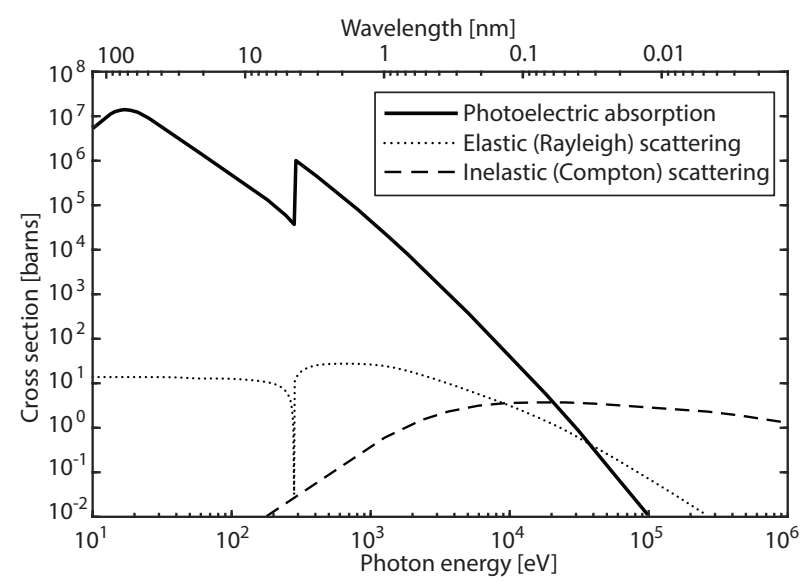

Figure 2.1. Atomic cross sections of carbon $\left(1\right.$ barn $\left.=10^{-24} \mathrm{~cm}^{2}\right)$. Photoelectric absorption is clearly the dominating proçess up to about $20 \mathrm{keV}$. Data from 2841 .

enough energy to ionize the atom, i.e., release the electron from its bound state. The new energy $E^{\prime}$ obtained by the photon, in the approximate case of a loosely bound electron, is given by $E^{\prime}=E /\left[1-\left(E / m_{e} c^{2}\right)(1-\cos \theta)\right]$, where $m_{e}$ is the electron mass and $c$ is the speed of light. The process of photoelectric absorption is predicted by classical electromagnetism, but better understood through the particle nature of light. Here, an incident photon is completely absorbed by an electron, which is typically ejected from its bound state.

The probabilities associated with the different interactions are usually described in terms of their respective cross sections, which are functions of the photon energy. As an example, the atomic cross sections of carbon are displayed in Fig. 2.1 for photon energies $10-10^{6} \mathrm{eV}$. For soft x-rays, photoelectric absorption is clearly the dominating interaction process. Here the photon energies match the inner-shell electron binding energies of many common elements (carbon, nitrogen, oxygen, etc.), making the interaction very probable. Compton scattering typically requires higher energies to occur and Rayleigh scattering is dominating at lower energies [41].

Absorption and elastic scattering can be modelled by considering the semi-classical interaction between an electromagnetic wave and bound electrons in atoms [40. From Maxwell's equations and the wave equation, light is shown to be a travelling wave of oscillating electric (E) and magnetic $(\mathbf{B})$ fields. In the special case of a wave of angular frequency $\omega$ travelling in free space, the electric field can be expressed in complex notation as

$$
\mathbf{E}_{\omega}(\mathbf{r}, t)=\mathbf{E}_{\omega}(0,0) e^{-i(\omega t-\mathbf{k} \cdot \mathbf{r})}
$$


where the wave vector $\mathbf{k}$ defines the direction of propagation.

When considering an electromagnetic wave travelling in matter, the electrons are included in the wave equation as point charges, held by the nucleus at discrete binding energies. (This is the non-classical part of the theory.) This leads to the derivation of the complex atomic form factor $f(\Delta \mathbf{k}, \omega)$ which describes the scattering amplitude as a function of the angular frequency and the change in the wave vector. Here we only consider scattering in the forward direction, and denote this part of the atomic form factor $f^{0}(\omega)=f_{1}^{0}(\omega)-i f_{2}^{0}(\omega)$. The complex refractive index can finally be defined as

$$
n(\omega)=1-\delta+i \beta=1-\frac{n_{a} r_{e} \lambda^{2}}{2 \pi}\left[f_{1}^{0}(\omega)-i f_{2}^{0}(\omega)\right]
$$

where $n_{a}$ is the atom density, $r_{e}=2.818 \cdot 10^{-15} \mathrm{~m}$ is the electron radius, and $\lambda$ is the wavelength in vacuum.

The complex refractive index turns up in the solution to the wave equation as a multiplicative factor to the wave vector. In the propagation direction of the wave, the electric field can be written as

$$
\mathbf{E}_{\omega}(\mathbf{r}, t)=\mathbf{E}_{\omega}(0,0) e^{-i \omega(t-r / c)} e^{-i(2 \pi \delta / \lambda) r} e^{-(2 \pi \beta / \lambda) r},
$$

where we have used the vacuum dispersion relation $k=2 \pi / \lambda=\omega / c$. It can now be seen that $\delta$ results in a phase shift of the electric field, while $\beta$ leads to a decrease of the field amplitude, i.e., absorption. Both these effects can be used as contrast mechanisms in microscopy and other types of imaging. Here, the main focus is absorption contrast.

The average intensity of the wave is obtained by taking the absolute square of the electric field, thus disregarding its time-dependent phase. The obtained expression is known as the Beer-Lambert law,

$$
I_{\omega}(\mathbf{r})=I_{\omega}(0) e^{-\mu r},
$$

where $\mu=4 \pi \beta / \lambda$ is called the local absorption coefficient (LAC).

\subsubsection{The water window}

As already mentioned, the dominating interaction process for soft x-rays is photoelectric absorption. The probability of this interaction depends on the matching of the photon energy to the binding energy of the electrons. When the photon energy reaches, or just surpasses, the binding energy of an inner electron the absorption cross section takes a discrete leap toward higher values [28]. This property is especially well-utilized as a contrast mechanism for biological imaging in the so-called water window [8]. The 


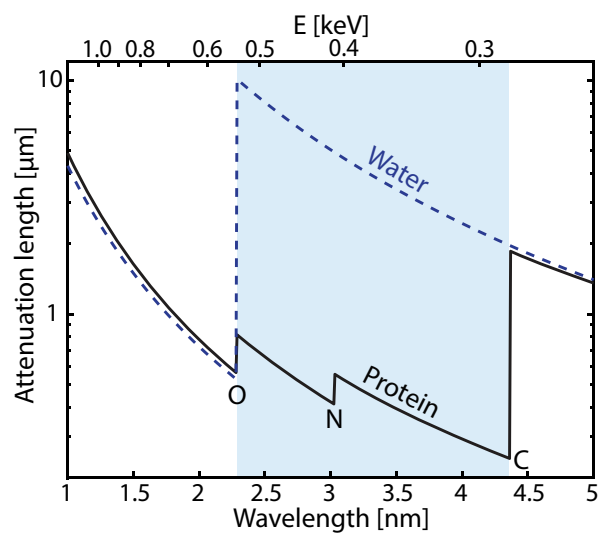

Figure 2.2. Soft $x$-ray attenuation in water and protein, respectively. The water window is indicated by the blue shaded area, and the K-absorption edges of carbon (C), nitrogen $(\mathrm{N})$ and oxygen $(\mathrm{O})$ are marked. A typical protein composition $\mathrm{H}_{86} \mathrm{C}_{52} \mathrm{~N}_{13} \mathrm{O}_{15} \mathrm{~S}$ and a density of $1.35 \mathrm{~g} / \mathrm{cm}^{2}$ were assumed 42. Data from 28.

K-edge of carbon, referring to the binding energy of its innermost electrons, is located at $\sim 290 \mathrm{eV}(\lambda \approx 4.3 \mathrm{~nm})$, while the K-edge of oxygen is located at $\sim 540 \mathrm{eV}(\lambda \approx 2.3 \mathrm{~nm})$. This results in a window, about $250 \mathrm{eV}$ wide, in the soft x-ray spectrum where the absorption is considerably higher in carbon than in oxygen. Consequently, absorption is high in carbon-dense biological materials, such as proteins and lipids, but significantly lower in water. Figure 2.2 illustrates the concept of the water window by plotting the attenuation (absorption) length in a typical protein and water, respectively.

\subsubsection{Soft $x$-ray reflectivity}

The refractive index also determines the behavior of an electromagnetic wave incident on a boundary between two materials. At the boundary the refractive index changes abruptly, which causes a part (or all) of the wave to be reflected, while the remaining wave continues into the material. This is described by the Fresnel equations [6], which depend on the polarization of the incident wave, i.e., the direction of the oscillating field compared to the boundary plane, and the refractive index of the two materials, $n_{1}$ and $n_{2}$. The amplitude reflection coefficients for a perpendicular $(\perp)$ and parallel (II) polarized wave are given by

$$
\begin{array}{r}
r_{\perp}=\frac{n_{1} \cos \phi_{1}-n_{2} \cos \phi_{2}}{n_{1} \cos \phi_{1}+n_{2} \cos \phi_{2}}, \\
r_{\|}=\frac{n_{2} \cos \phi_{1}-n_{1} \cos \phi_{2}}{n_{2} \cos \phi_{1}+n_{1} \cos \phi_{2}},
\end{array}
$$


where $\phi_{1}$ is the incident angle from the surface normal and $\phi_{2}$ is the transmission angle, given by Snell's law

$$
n_{1} \sin \phi_{1}=n_{2} \sin \phi_{2} .
$$

While Eqs. 2.5 and 2.6 relate the incident and reflected field amplitudes, the reflectivity $\left(R=|r|^{2}\right)$ relates the intensity of the waves. For soft $\mathrm{x}$ rays, the refractive index is very close to unity. This gives both a very small refraction, from Eq. 2.7 and very low reflectivity, especially at low incidence angles. As an example, a $\lambda=2.48 \mathrm{~nm}$ wave at normal incidence on a gold surface gives a reflectivity on the order of $10^{-7}-10^{-6}$ [28].

\subsection{Image formation in 2D}

All electromagnetic radiation will, as a consequence of its wave property, be subject to scattering by diffraction [43]. This phenomenon is defined as the deviation of the wave from its otherwise straight, or rectilinear, path as it encounters an edge where the index of refraction suddenly changes. This results, e.g., in the bending of light around a corner and the spread of light that travels through an aperture. The latter example is of fundamental importance to the theory of image formation, since apertures, or equivalently lenses and mirrors, are the main components of most imaging systems. This section summarizes the theory of image formation, based on the theory of classical electromagnetism and using the tools of Fourier optics.

\subsubsection{Projection imaging}

We start at the simple image formation model called projection imaging. This theory discards the wave nature of light and only considers rays travelling in the forward direction. Nevertheless, some parts of the theory are relevant for the later discussions.

Consider a ray propagating through a medium in the positive $z$-direction, from point $A$ to point $B$. Using the notation of the Beer-Lambert law (Eq. 2.4), and allowing the LAC $\mu$ to vary in space, the intensity can be written as

$$
I_{B}(x, y)=I_{A}(x, y) e^{-\int_{A}^{B} \mu(x, y, z) d z} .
$$

In this model of image formation, the rays exiting the object continue in a straight path toward the detector. Assuming that all the rays originate from the same point, this gives a shadow image $I_{D}$ of the object with a magnification $M$, which is determined by the distances to the object and

the detector, 


$$
I_{D}(M x, M y)=\frac{1}{M^{2}} I_{B}(x, y) .
$$

No source is infinitely small and this fact will inevitably lead to a smearing of the image, which depends on the size of the source. This is called the penumbra effect and should not be confused with the spread of light due to diffraction. At high magnifications $(M \gg 1)$, the smearing in the object plane will have the same size as the source. For this reason, high-resolution microscopy $(<100 \mathrm{~nm})$ is rarely attempted using simple projection imaging 44,45$]$.

\subsubsection{Imaging by a lens}

Fourier optics provides the necessary tools to describe imaging by lenses, while taking into account the wave property of light that leads to diffraction. The foundation of Fourier optics is the concept of the plane wave spectrum, in which each point on the wave front is described as a continuous superposition of monochromatic plane waves [43. These are natural solutions to the wave equation, (where matter is assumed to be isotropic, non-magnetic and homogeneous), and the assumption of linearity allows separation of the electric field into time-harmonics.

The mathematical foundation of Fourier optics was first developed by Gustav Kirchhoff [46 in the 1880s and Arnold Sommerfeld [47] in the 1890s. Their theory showed that diffraction of a plane wave through an aperture $P(x, y)$, at a far distance $z$, results in a scaled Fourier transform of the aperture. This is known as Fraunhofer diffraction and can be written as

$$
\begin{aligned}
& h(x, y)=e^{i \frac{k}{2 z}\left(x^{2}+y^{2}\right)} \mathcal{F}\{P(\lambda z \xi, \lambda z \eta)\}= \\
& =e^{i \frac{k}{2 z}\left(x^{2}+y^{2}\right)} \iint_{-\infty}^{\infty} P(\lambda z \xi, \lambda z \eta) e^{-i 2 \pi(x \xi+y \eta)} d \xi d \eta,
\end{aligned}
$$

where the amplitude of the wave has been chosen to normalize the expression, for simplicity. The factor outside the integral is then simply a multiplicative phase. Equation 2.10 is valid provided that $z>\frac{2 D^{2}}{\lambda}$, where $D$ is the aperture diameter and $\lambda$ is the wavelength. This regime is often referred to as the far field [43]. A fundamental property of the Fourier transform is that a function that extends far in space gives a narrow Fourier transform, while a narrow function gives a wide Fourier transform. This means that the smaller an aperture is, the larger the spread due to diffraction will be.

Building on the theory of diffraction through an aperture, imaging through an ideal, i.e., aberration-free, lens can be derived for the simple case of a monochromatic plane wave. It turns out that this can be 
described as the convolution between the perfect image, given by the lens equation of geometrical optics, and the Fraunhofer diffraction pattern of the exit pupil. The electric field in the image plane can be written as

$$
E_{i}(x, y)=\frac{1}{M} h(x, y) \otimes E_{o b j}\left(\frac{x}{M}, \frac{y}{M}\right)
$$

where $E_{o b j}$ is the field exiting the object, $M$ is the magnification and $h(x, y)$ is given by Eq. 2.10 It can now be seen that the function $h(x, y)$ will act as a point-spread function to the system. This theory can be extended to polychromatic non-plane waves through the previously mentioned assumption of linearity. At this point, however, we must consider two separate imaging cases: coherent and incoherent imaging.

\section{Coherent imaging}

Coherence in this case refers to the spatial, or transverse, coherence that depends largely on the physical extension of the source. Simply put, if all incident waves originate from the same point, the field that reaches the plane of an aperture or a lens will be known across the whole plane, given the field in one point. This is the case of fully coherent illumination. For this treatment to be valid, quasimonochromatic illumination is assumed, meaning that there is a finite but small spectral bandwidth. Detailed discussions on this concept are presented in textbooks 43, 48, 49.

From these assumptions it turns out that Eq. 2.11 is still valid in the case of coherent imaging. Furthermore, this can be conveniently formulated by using Fourier transforms, and thereby describing light from the object in terms of its spatial frequencies $\left(\nu_{X}, \nu_{Y}\right)$. By taking the Fourier transform of Eq. 2.11 it is shown that coherent imaging is a simple filtering of spatial frequencies in Fourier space. This can be written as

$$
I_{i}(x, y)=\frac{1}{M^{2}}\left|\mathcal{F}^{-1}\left\{\mathcal{F}\left\{E_{o b j}\left(\frac{x}{M}, \frac{y}{M}\right)\right\} \times \tilde{P}\left(\nu_{X}, \nu_{Y}\right)\right\}\right|^{2},
$$

where $\tilde{P}$ is the scaled exit aperture of the lens, defined by $\tilde{P}(x, y)=P\left(\lambda z_{i} x, \lambda z_{i} y\right)$, and $z_{i}$ is the image distance. The most common case of a circular lens can thus be described by the filtering function

$$
\tilde{P}(\nu)=\operatorname{circ}\left(\frac{\nu}{D / 2 \lambda z_{i}}\right)= \begin{cases}1, & \nu \leq \frac{D}{2 \lambda z_{i}} \\ 0, & \nu>\frac{D}{2 \lambda z_{i}}\end{cases}
$$

where $D$ is the lens diameter and $\nu=\sqrt{\nu_{X}^{2}+\nu_{Y}^{2}}$. 


\section{Incoherent imaging}

The case of fully incoherent imaging is usually more applicable to real imaging scenarios. This can also be described as a filtering in Fourier space, but of the intensity rather than the electric field,

$$
I_{i}(x, y)=\frac{1}{M^{2}} \mathcal{F}^{-1}\left\{\mathcal{F}\left\{I_{o b j}\left(\frac{x}{M}, \frac{y}{M}\right)\right\} \times O T F\left(\nu_{X}, \nu_{Y}\right)\right\}
$$

The filtering in this case is done by the optical transfer function (OTF), which can be calculated as the scaled auto-correlation of the aperture. For imaging through a circular lens, the OTF takes the form

$$
O T F(\nu)=\left\{\begin{array}{cl}
\frac{2}{\pi}\left[\arccos \left(\frac{\nu}{D / \lambda z_{i}}\right)-\frac{\nu}{D / \lambda z_{i}} \sqrt{1-\left(\frac{\nu}{D / \lambda z_{i}}\right)^{2}}\right], & \nu \leq \frac{D}{\lambda z_{i}} \\
0, & \nu>\frac{D}{\lambda z_{i}}
\end{array}\right.
$$

Since the OTF can, in some cases, reach negative values it is common to consider its absolute value $|O T F(\nu)|$. This is known as the modulation transfer function (MTF).

\section{Resolution in coherent and incoherent imaging}

The images produced by an imaging system can look very different in the coherent and incoherent case. A comparison, using simulated images of a Siemens star pattern, is given in Fig 2.3. One of the most notable effects of coherent imaging (Fig. 2.3.a)) is the ringing that occurs at sharp edges [43]. Instead of falling off gradually, like in the incoherent case (Fig 2.3 (b)), the image intensity will oscillate close to the edge and then fall off more abruptly. In some cases this edge enhancement can be an advantage, while in other cases it will only disturb the image, since the ringing effect is difficult to separate from other contrast mechanisms.

Another difference between coherent and incoherent imaging is the highest attainable resolution. To illustrate this, the modulation transfer in the two cases, given by Eqs. 2.12 and 2.14 is shown in Fig. 2.3. (c). It can be seen that the cut-off frequency, i.e., the frequency where the modulation transfer drops to zero, is twice as high for the incoherent case. In theory, incoherent imaging can thus achieve twice as high resolution compared to coherent imaging. However, in the coherent case the modulation transfer is unity all the way to the cut-off frequency and will thus produce better contrast.

Even though the modulation transfer as a function of spatial frequency provides more complete information about the resolution, it is instructive to 
(a)
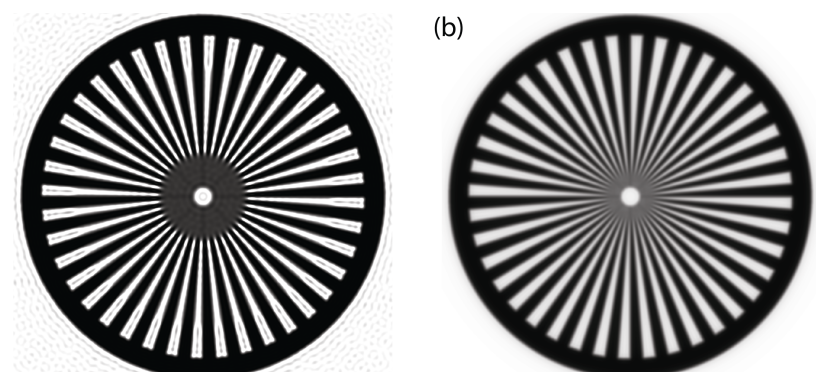

(c)

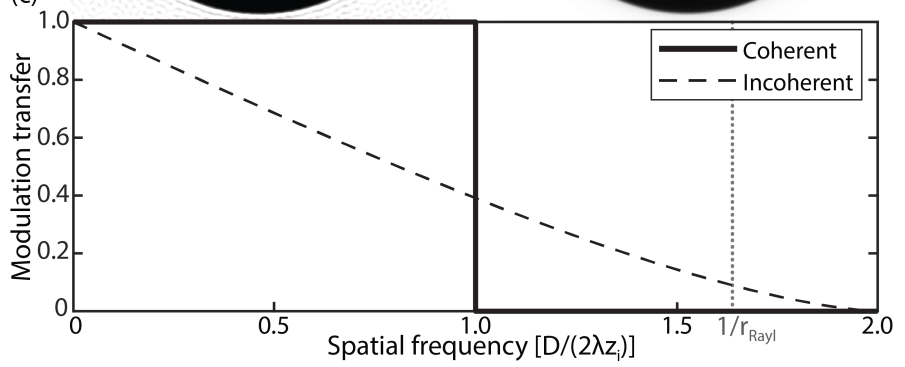

Figure 2.3. Comparison between (a) coherent and (b) incoherent imaging of a Siemens star test pattern. (c) Plot of the modulation transfer in the coherent and incoherent case, for a circular lens. The spatial frequency (in the image plane) is given in multiples of $D /\left(2 \lambda z_{i}\right)$, and the classical Rayleigh resolution limit is indicated at $1 / r_{\text {Rayl }}$.

compare this to the classical Rayleigh resolution criterion (Fig. 2.4). Here, two incoherent point sources of wavelength $\lambda$, separated by a distance $r$, are imaged by a circular lens of diameter $D$ and focal length $f$. According to the Rayleigh criterion, the two point sources can be resolved if

$$
r \geq r_{\text {Rayl }}=1.22 \frac{f \lambda}{D}=0.61 \frac{\lambda}{N A},
$$

where the numerical aperture is defined by $N A=D /(2 f)$. The factor 1.22 is given by the definition that the first minimum of the diffraction pattern from one point source should coincide with the center of the other point source. Equivalently, there should be at least a $26.3 \%$ dip in intensity between the two maxima. Note the resemblance to Eq. 1.1, in which Abbe used a factor 1 instead of 1.22. Note also that this discussion is carried out in the object plane. The result can easily be transferred to the image plane by using $z_{i} \approx M f$, which is true for the large magnifications used in microscopy. In order to relate this definition of resolution to the modulation transfer for coherent and incoherent imaging, the Rayleigh resolution limit is included in Fig. 2.3 (c), at spatial frequency $1 / r_{\text {Rayl }}$. 


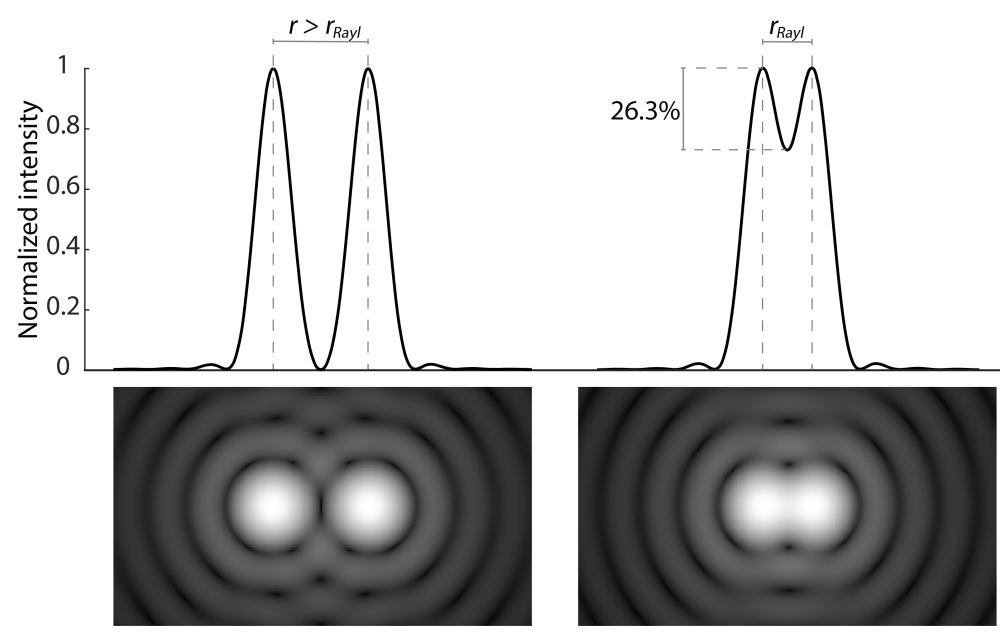

Figure 2.4. Illustration of the Rayleigh resolution criterion for two incoherent point sources. When the separation $r \geq r_{\text {Rayl }}$, the points are said to be resolved.

Finally, it should be noted that coherent and incoherent imaging are extreme cases. Many imaging systems, like soft x-ray microscopes, can be described as being partially coherent, meaning that they experience some coherence effects. More details on the theory of partially coherent imaging can be found in 48,50 .

\subsubsection{Coherent diffraction imaging}

When coherent illumination is available it is possible to do coherent diffraction imaging (CDI), in which the diffraction pattern is used directly in the far field, without involving lenses. In theory it is possible to reconstruct an image from its complex Fraunhofer diffraction pattern (Eq. 2.10) by using the inverse Fourier transform. In reality, however, no detector is fast enough to detect the complex electric field due to the rapidly varying phase. This gives rise to the so-called phase problem. Instead, the field intensity is registered and used together with iterative computation algorithms and smart initial constraints in order to reconstruct an image of the object. CDI and related techniques, such as ptychography and x-ray holography, are currently used with coherent $\mathrm{x}$-ray sources in a wide range of applications 36, 51]. Nevertheless, this Thesis deals mainly with incoherent (and partially coherent) x-ray microscopy and therefore these methods will not be further discussed. 


\subsection{Tomographic (3D) image formation}

The full potential of soft x-ray imaging can be utilized by performing computed tomography $(\mathrm{CT})$, thereby obtaining a complete 3D image of the object. CT is widely used today as a medical imaging method for diagnostic purposes, in medical and biological research, as well as in material science. The concept has been extensively examined and is described in numerous textbooks $[52,53]$. CT typically refers to tomographic imaging using $\mathrm{x}$-rays, even though the same concept is used with other imaging techniques like TEM, where it is commonly referred to as electron tomography (ET).

The mathematical foundations of tomography were derived by Radon long before computers existed to enable any real tomographic imaging [54]. The theory is most conveniently described for the case of a $2 \mathrm{D}$ object, giving $1 \mathrm{D}$ projections. This is then easily generalized to the $3 \mathrm{D}$ case by considering many independent 2D slices, which stacked together make up a 3D volume. The basis of the mathematical formulation by Radon is the so-called Radon transform, which describes the projection of a finite $2 \mathrm{D}$ object, with density distribution $\mu(x, y)$, at an angle $\theta$ around the z-axis,

$$
T_{\theta}(u)=\int_{L(\theta)} \mu(x, y) d l .
$$

The line $L(\theta)$ along which the projection is acquired is defined by $(x, y)=$ $(u \cos \theta+l \sin \theta,-u \sin \theta+l \cos \theta)$. The main result by Radon [54] states that the original density distribution $\mu(x, y)$ can be uniquely determined from $T_{\theta}(u)$, assuming continuous (infinite) projections. Even though an infinite number of projections is obviously not feasible, there are various techniques to reconstruct something very close to the original object. The finite set of projections, in the case of a $2 \mathrm{D}$ object, is usually referred to as a sinogram. In the extended 3D case it is instead called a tomogram.

The projections given by the Radon transform (Eq. 2.17) are closely related to simple projection imaging, described by Eq. 2.8. It can be seen that $T_{\theta}$ is obtained by normalizing the right-hand side of Eq. 2.8 and taking the negative logarithm $(-\log )$. The density function $\mu(x, y)$ is, in this case, taken to be the LAC (hence the similar notation). In this discussion, the actual imaging step is omitted and a magnification of $M=1$ is assumed, for simplicity.

\subsubsection{The Fourier slice theorem}

The theory of tomographic (3D) image formation can be neatly described using the Fourier slice theorem. It states that the 1D Fourier transform of the projection of a $2 \mathrm{D}$ object is equal to a line in the $2 \mathrm{D}$ Fourier transform 


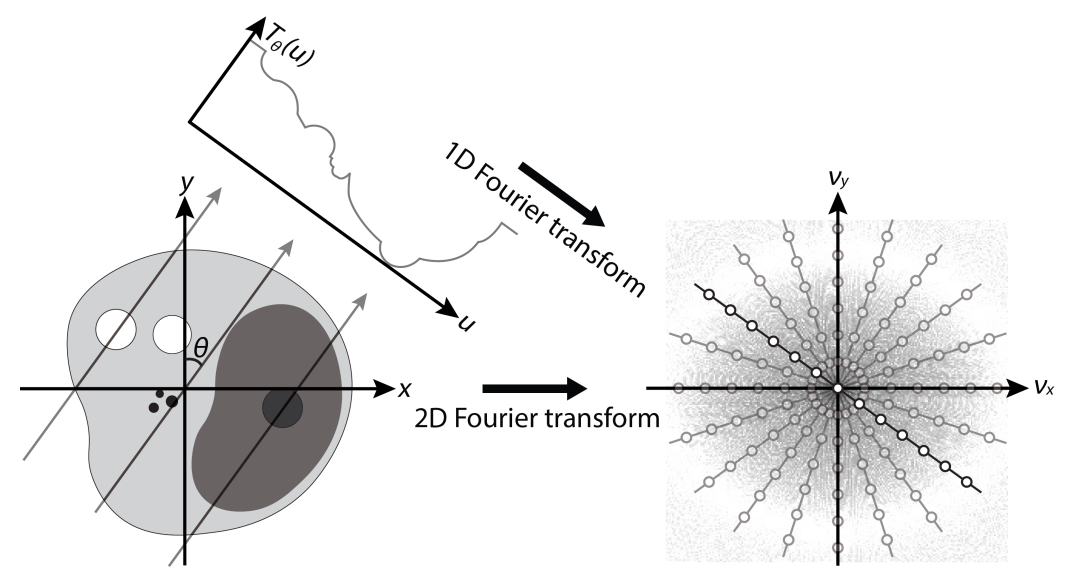

Figure 2.5. Illustration of the Fourier slice theorem. A projection image of the 2D object, when Fourier transformed, produces a line in the 2D Fourier transform of the object. By gradually rotating and acquiring projection images, information about the full Fourier transform of the object can be obtained.

of the object itself. This process is visualized in Fig. 2.5. The Fourier slice theorem can be readily derived by taking the Fourier transform of Eq. 2.17 and comparing with the Fourier transform of $\mu(x, y)[52$. Just as in our previous discussion this theory can easily be extended to $3 \mathrm{D}$ objects.

\subsubsection{Tomographic reconstruction}

It is possible to use the result of the Fourier slice theorem to directly reconstruct the object from its Fourier transform (cf. Fig 2.5). This method is, however, rarely used since it involves resampling the obtained Fourier transform from the sampled points along concentric circles to, e.g., Cartesian coordinated, before the inverse Fourier transform can be taken 52 .

The most commonly used reconstruction technique is called filtered back-projection. Here, the projections are back-projected one by one onto the object volume, along the same angle as they were acquired. Prior to the back-projection, the projections are filtered in Fourier space to decrease the contribution from low spatial frequencies. The whole process of computing a reconstruction, $R(x, y)$, of the object can be written as

$$
R(x, y)=\sum_{\theta} \mathcal{F}^{-1}\left\{P\left(\nu_{u}\right) \times \mathcal{F}\left\{T_{\theta}(u)\right\}\right\},
$$

where $P\left(\nu_{u}\right)$ is the filter function in Fourier space and $T_{\theta}(u)$ is given by Eq. 2.17. 
The reconstruction problem can also be formulated as a large system of linear equations, where each variable corresponds to a pixel (2D) or a voxel (3D) in the reconstructed object. This is the basic principle of the algebraic reconstruction technique (ART) [55]. One of the main challenges in solving the linear equations is the fact that they are, in most realistic cases, underdetermined [52]. To this problem one must add the inconsistencies that arise due to noise, as well as the fact that the number of equations becomes very large for high-resolution acquisitions at many different angles. The set of equations must instead be made into an optimization problem, which is solved iteratively until the estimated error becomes acceptably small. The error is given by the difference between the calculated forward projection, using the current pixel or voxel values, and the acquired projection images. The method used to adjust the pixel values between iterations varies between different algorithms (ART, SIRT, SART etc.) [52,56].

\subsubsection{Resolution in tomography}

Building on the theory of the Fourier slice theorem, it is possible to estimate the achievable resolution of the tomographic reconstruction. The sampling in Fourier space will be determined by the resolution in each projection, as well as the spacing of projection angles. For this discussion, assume that projections are acquired over a $0^{\circ}-180^{\circ}$ range and that the resolution in the individual projections is not limiting. The highest sampling density is obtained at low spatial frequencies (thus the need for filtering in the FBP reconstruction algorithm). Toward the higher spatial frequencies, the distance between the sampled points gradually increase, as depicted in Fig. 2.5 The highest resolvable spatial frequency $\nu_{c}$, or equivalently, the smallest resolvable period $\Delta x$ can be derived from the spacing between the outermost points in the sampled Fourier spectrum, according to 57

$$
\Delta x=\frac{1}{\nu_{c}} \geq \frac{\pi D}{N}
$$

where $D$ is the object diameter and $N$ is the number of projection angles. This is known as the Crowther criterion.

The Crowther criterion provides a good estimation of the number of projection angles needed in the tomographic image acquisition. Nevertheless, it describes an ideal case. In many cases the full $0^{\circ}-180^{\circ}$ range is not available during tomographic imaging. This leads to a missing wedge in the sampled data and subsequent artifacts in the reconstructed image. Exactly how the resolution is affected is difficult to describe, since it depends on the reconstruction algorithm. There are methods to reduce artifacts from the missing wedge. Since this is a matter of missing information these methods 
either include acquiring more information, like tilt around another axis (dual tilt [58]), or putting additional constraints on the algebraic reconstruction algorithms [59,60].

In soft x-ray microscopy (both scanning and full-field), the depth of focus (DOF) typically puts additional limitations on the tomographic resolution. If the imaged object is significantly larger than the DOF, the outer parts of the object will be blurred in the images and information will be lost. A possible solution to this problem is given by the focus-stack back-projection (FSBP) algorithm 61. Here, projections at different defocus positions, for each tilt angle, are combined in order to minimize the blurring. The DOF of soft x-ray optics is further discussed in Chapter 3 


\section{Chapter 3}

\section{Soft x-ray microscopy}

The main focus of this Thesis is lens-based soft x-ray microscopy, in which soft x-rays and specialized optics are used to form real images of the sample. This technique much resembles visible-light microscopy in its arrangement, but due to the nature of soft x-ray interaction with matter (cf. Chapter 2 ) most components are fundamentally different. This Chapter describes the general arrangement of the soft x-ray microscope and its main components, from x-ray sources and optics, to sample environment and x-ray detection.

\subsection{Scanning or full-field}

As mentioned in Chapter 1, lens-based soft x-ray microscopy is either done in full-field, where the entire sample is illuminated and imaged simultaneously, or in a scanning arrangement. The full-field arrangement, often referred to as full-field transmission x-ray microscopy (TXM), has the closest resemblance to the original light microscope. Here, a condenser collects the light from the source to illuminate the sample and a high-resolution objective lens creates a magnified real image on the detector. This arrangement is depicted in Fig. 3.1 (a). The resolution in TXM, in the case where aberrations and system stability are not limiting, is set by the resolving power of the objective $[50]$.

In the scanning arrangement (Fig. 3.1 (b)), a high-resolution zone plate lens (cf. Section 3.3.3 is used to focus the x-rays from the source onto a small spot on the sample. The transmitted x-rays are registered by the detector and the sample is moved to the next position where the process is repeated. Here, the size of the focus spot from the zone plate determines the resolution. In order to achieve a diffraction limited spot, the x-ray source needs to be spatially coherent 32,62 . Alternatively, a small pinhole 


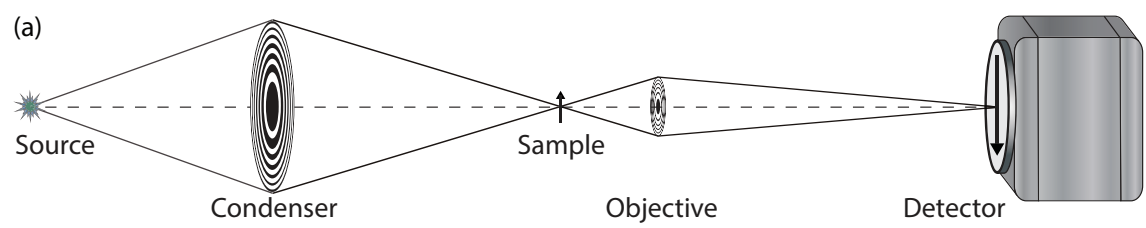

(b)

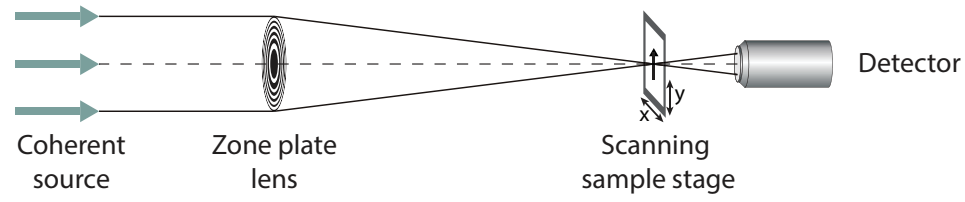

Figure 3.1. (a) The arrangement of a full-field transmission x-ray microscope (TXM), consisting of an extended x-ray source, a condenser, an objective and a detector. The condenser and objective are depicted as Fresnel zone plates, even though other types of optics are available. (b) The arrangement of a scanning transmission x-ray microscope (STXM). Here, the coherent x-rays are focused onto a small spot on the sample, which is placed on a scanning stage.

can be used to decrease the spot size [31], which unfortunately also means decreasing the photon flux.

The Stockholm laboratory x-ray microscope, described in Chapter 4 is a full-field transmission soft x-ray microscope, based on an incoherent (or partially coherent) compact x-ray source. Since the experimental work in this Thesis was mainly done using this setup, the full-field arrangement will be discussed in more detail.

\subsection{Soft x-ray sources}

To enable microscopy, the x-ray source needs to deliver a high photon flux from a sufficiently small area, and with the appropriate spectral properties. These properties are often summarized using a figure of merit called spectral brightness (sometimes brilliance or just brightness), defined as photons $/\left(\mathrm{s} \times \mathrm{sr} \times \mu \mathrm{m}^{2} \times 0.1 \% \mathrm{BW}\right)$. It generally gives a good idea of the flux of photons available for imaging, by including the solid angle of emission (sr), the size of the source $\left(\mu \mathrm{m}^{2}\right)$, and by restricting the spectral bandwidth $(\mathrm{BW}=\Delta \lambda / \lambda)$ to $0.1 \%$. However, the spectral brightness does not always provide the full picture, and should therefore only be used as a starting point for more detailed discussions. Examples of this include achromatic systems, where a limited BW is not favorable, and arrangements where an even smaller source would limit the field of view (FOV). It should also be noted that the degree of spatial coherence is generally of major concern. 

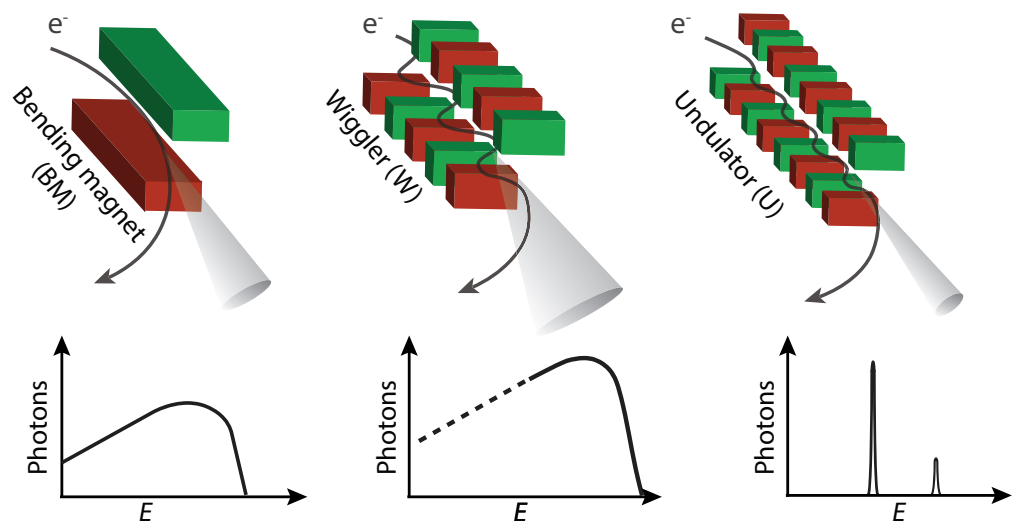

Figure 3.2. Synchrotron radiation sources and their typical radiation spectra.

\subsubsection{Synchrotron sources}

According to classical electromagnetism, an oscillating charged particle will emit electromagnetic waves. The radiated power will depend on the emission angle as $\sin ^{2} \theta$, known as the electric dipole radiation pattern [40]. By accelerating the oscillating particle to relativistic speeds (close to the speed of light), the radiation will instead be emitted in a narrow cone around the travelling direction of the particle, thereby increasing the brightness of the emission by many orders of magnitude. This is the basic principle of synchrotron radiation [63], utilized at many large-scale facilities around the world. The synchrotron-radiation sources are characterized by high brightness, high versatility and the possibility to tune both spatial coherence and spectral properties.

At a synchrotron-radiation facility, electrons are accelerated and injected into a large storage ring, often with a circumference of several hundred meters. At modern facilities, the storage ring consists of many long straight sections. Insertion devices, consisting of periodic magnetic structures, are placed in these straight sections to make the electrons oscillate and thereby emit radiation. The insertion devices are usually divided into two classes, called undulators $(\mathrm{U})$ and wigglers $(\mathrm{W})$, depending on their period and magnetic field strength. In between the straight sections, the electrons are guided by the magnetic field of bending magnets (BM), which also cause the electrons to radiate. Figure 3.2 illustrates the working principle of synchrotron sources, as well as their typical emission spectra. 


\section{Bending magnets}

The radiation from a bending magnet is characterized by a broad spectrum and moderate x-ray flux, compared to the insertion devices. The critical wavelength, $\lambda_{c}$, which defines the center of the emitted power, is given by 63

$$
\lambda_{c}=\frac{4 \pi m c}{3 e B \gamma^{2}},
$$

where $e$ and $m$ are the charge and the mass of the electron, $B$ is the magnetic field and $c$ is the speed of light. $\gamma$ is the Lorentz factor from special relativity, defined by $\gamma=\sqrt{\frac{1}{1-v^{2} / c^{2}}}$, where $v$ is the speed of the electrons. This factor also determines the width of the emission cone. The half angle of the emission is given by

$$
\theta \approx \frac{1}{2 \gamma}
$$

\section{Undulators and wigglers}

The insertion devices will cause the electrons to undergo transverse oscillations as they travel through a periodic magnetic field. A mathematical treatment, starting with the force equation of a charged particle, shows that the nature of the oscillations depends greatly on the amplitude $B_{0}$ and pe$\operatorname{riod} \lambda_{u}$ of the magnetic field. It is convenient to define the non-dimensional magnetic deflection parameter as

$$
K=\frac{e B_{0} \lambda_{u}}{2 \pi m c} .
$$

For $\mathrm{K}<1$, the magnetic force is relatively weak, and the electrons will therefore experience close-to harmonic oscillations. Insertion devices with this property are referred to as undulators. In the reference frame of the electrons the emitted radiation will approximately follow the $\sin ^{2} \theta$ pattern of an electric dipole. Furthermore, the spectral bandwidth is determined by the number of undulator periods $N$, according to

$$
\frac{\Delta \lambda}{\lambda}=\frac{1}{N}
$$

Due to relativistic length contraction, the emission will be focused in the forward direction of the electrons and the radiated wavelength in the laboratory frame will be much shorter than the undulator period, reaching far into the x-ray regime. There will also be a broadening in the emission 
spectrum, arising from the relativistic Doppler effect. However, this broadening will only affect off-axis emission, and can thus be removed by filtering the emission beam through a pinhole. Specifically, it can be shown that the original spectral bandwidth, given by Eq. 3.4 is recovered for a central emission cone of half angle $\theta_{c e n}=1 /(\gamma \sqrt{N})$.

The wavelength of the emitted radiation is given by the undulator equation,

$$
\lambda=\frac{\lambda_{u}}{2 \gamma^{2}}\left(1+\frac{K^{2}}{2}+\gamma^{2} \theta^{2}\right),
$$

where $\theta=0$ gives the emission in the forward direction. It can be seen that tuning of the wavelength can be done by adjusting the Lorentz factor $\gamma$, or more conveniently, the magnetic deflection parameter $K$.

As $K$ reaches and exceeds unity, the higher harmonic oscillations, both transverse and axial, become significant. Insertion devices for which $K \gg 1$ are called wigglers. Here, the numerous high harmonics result in tightly spaced emission peaks, which eventually merge into a continuum. The wigglers are typically characterized by high photon flux and high photon energies.

\subsubsection{Free-electron lasers}

Closely related to the synchrotron sources are the x-ray free-electron lasers (FEL), which use linear accelerators in combination with very long undulators to achieve even higher photon flux and spatial coherence 64]. As the electrons travel through the long undulator, they start interacting with the radiated field through the Lorentz force, causing them to modify their propagation speed. Depending on the phase of the oscillating electron, relative to the field, it will either speed up or slow down slightly, eventually leading to ordering of the electrons into so-called microbunches. The ordered electrons will then radiate in phase with each other, thus adding the radiation amplitude rather than the intensity. The total photon flux will thus be proportional to the square of the number of electrons $N_{e}^{2}$, instead of just $N_{e}$ as in conventional undulators.

Around 7 x-ray FEL facilities are currently in operation around the world 65, 66. The applications mainly include experiments that utilize the high coherence and ultra-short pulses available. It is, e.g., possible to collect snapshots of (the diffraction pattern from) protein molecules, using $\mathrm{x}$-ray doses that completely destroy samples, by so-called diffraction before destruction 67]. Since X-ray FELs are not used for lens-based XRM, they will not be further discussed here. 


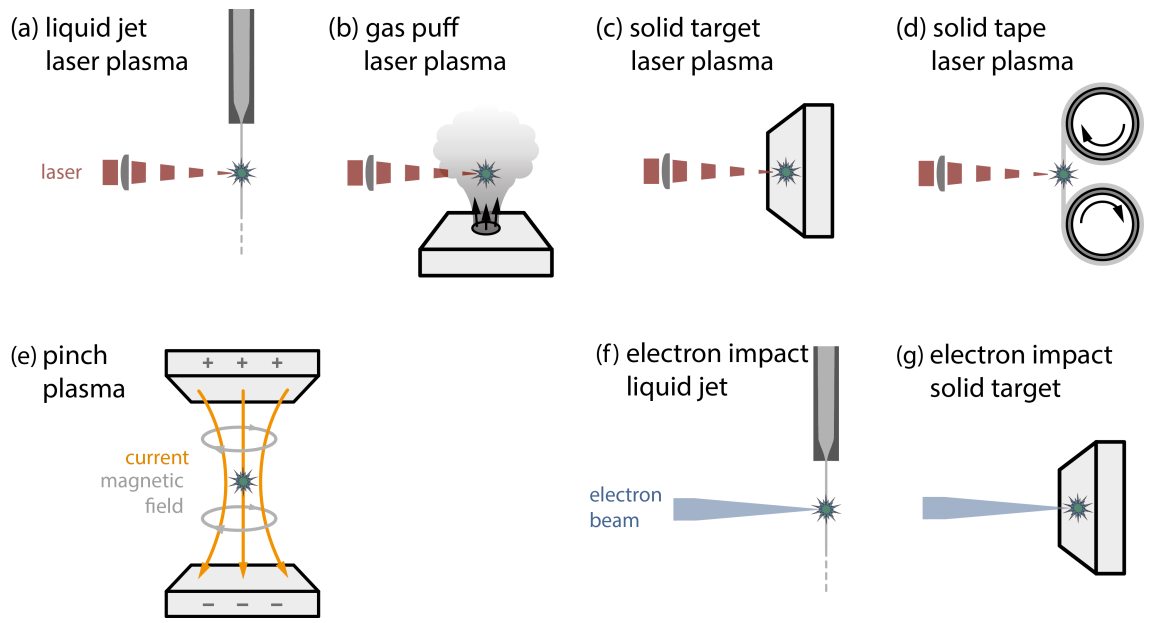

Figure 3.3. Laboratory soft x-ray sources. (a)-(d) Laser-plasma sources with various targets. (e) Discharge, or pinch, plasma source. (f)-(g) Electron impact sources. (Figure adapted from Paper E)

\subsubsection{Laboratory $x$-ray sources}

Laboratory, or compact, sources for x-ray microscopy have been developed over the past few decades and are now reaching a point where they can provide an alternative to accelerator-based sources for certain applications. A detailed comparison of laboratory x-ray sources for TXM is given in Paper E. Only a brief summary is given here.

\section{Discharge sources}

The first laboratory TXM, developed by Schmahl et al. [68, was based on a nitrogen-gas discharge source [69]. In discharge $\mathrm{x}$-ray sources a gas is typically injected into the space between an anode and a cathode, where an electric spark is used to create a plasma. The plasma electrons accelerate rapidly toward the anode while emitting x-rays. In doing so, they also create a magnetic field that pulls the electrons toward the central axis, thus decreasing the size of the emission spot and increasing the plasma temperature. This phenomenon is known as a z-pinch [70].

The $\mathrm{x}$-ray emission from discharge $\mathrm{x}$-ray sources consists of a broad spectrum, with some additional element-specific peaks that depend on the target gas. In the soft x-ray regime, the broadband emission can be described, with reasonable accuracy, as blackbody radiation [71]. This means that the intensity will peak at a wavelength given by Wien's displacement 
law

$$
\lambda_{\text {peak }}=\frac{2.898 \cdot 10^{6} \mathrm{~nm} \cdot \mathrm{K}}{T},
$$

where $T$ is the plasma temperature. When the broadband emission overlaps with the characteristic electron transition energies of the target, the probability of these transitions increase, and the line emission becomes more intense.

Modern discharge x-ray sources have been demonstrated in TXM 72, 73], with spectral brightnesses of up to $2.4 \cdot 10^{9}$ photons $/\left(\mathrm{s} \times \mathrm{sr} \times \mu \mathrm{m}^{2} \times 0.1 \% \mathrm{BW}\right)$ for $\lambda=2.88 \mathrm{~nm}$.

\section{Laser-plasma sources}

The emission from laser-plasma x-ray sources can be described using Wien's displacement law and element-specific line emission, following the same principles as for discharge sources. In this case, the plasma is created by focusing a pulsed high-power laser onto a target material. The conversion efficiency from laser energy to x-rays is typically on the order of tens of percent, depending on the target material and laser wavelength [74]. Combining this conversion efficiency with the Stefan-Boltzmann law for blackbody radiation, a relation can be derived between the incident laser intensity and the plasma temperature. Using Eq. 3.6 this can give a good estimation of the emitted spectrum. As an example, laser intensities on the order of $10^{13}-10^{14} \mathrm{~W} / \mathrm{cm}^{2}$ can be estimated to produce strong emission in the soft $\mathrm{x}$-ray regime $(\lambda \approx 0.5-5 \mathrm{~nm})$.

Laboratory x-ray microscopy has been demonstrated using laser-plasma sources with various types of targets, such as solid bulk $75-77$, solid tape [78], liquid jet [79, 80], and gas-puff targets 81,82 . Out of these, the liquid-jet targets have demonstrated the highest spectral brightness $\left(>10^{12}\right.$ photons $/\left(\mathrm{s} \times \mathrm{sr} \times \mu^{2} \times 0.1 \% \mathrm{BW}\right)$ for $\left.\lambda=2.48 \mathrm{~nm}\right)$ 83. Furthermore, the liquid-jet and gas-puff targets have the important advantage of being regenerative and relatively debris-free $[79,82,84$. .

\section{Electron-impact sources}

In the original x-ray tube, used by Röntgen in his early experiments, x-rays were generated by letting high-speed electrons collide with a metal anode. This principle is still used in modern versions of the x-ray tube, which are found, e.g., in diagnostic x-ray imaging modalities at hospitals and clinics. High-Z anode materials such as tungsten or rhodium are commonly used, which mainly generate bremsstrahlung x-rays with typical peak photon energies on the order of tens of $\mathrm{keV}$ (hard x-rays) 53 . 
For soft x-ray generation, the bremsstrahlung emission is typically very weak compared to $K_{\alpha}$ line emission [85. On the other hand, the nonradiative Auger process takes up most of the incident energy, making the total x-ray conversion efficiency low 86.

Electron impact soft x-ray sources have been used for microscopy purposes, but generally with low brightness $87-89$. Here, various solid targets were used. A higher-brightness electron-impact source, using a water-jet target, was developed by Skoglund et al. 90]. It produced a brightness of $5 \cdot 10^{8}$ photons $/\left(\mathrm{s} \times \mathrm{sr} \times \mu \mathrm{m}^{2} \times 0.1 \% \mathrm{BW}\right)$ at $\lambda=2.36 \mathrm{~nm}(E=525 \mathrm{eV})$, but was never used for microscopy.

\section{Coherent sources}

A number of different techniques can be used to produce high-brightness coherent illumination on a laboratory scale. These include high harmonic generation (HHG) 91, 92, plasma-based x-ray lasers (XRL) 93, 94 and various techniques based on laser-wakefield accelerators (LWFA) 95]. Microscopic imaging in the EUV 92,96 and hard x-ray regime 95$]$ has been demonstrated. However, soft x-ray microscopy on a laboratory scale is currently not feasible [91,97]. Furthermore, the coherent sources are better suited for CDI methods than for lens-based microscopy and are therefore outside the scope of this Thesis.

\subsection{Soft x-ray optics}

As discussed in Chapter 2 the refractive index, $n=1-\delta+i \beta$, is very close to unity for soft x-rays, and furthermore, the absorption is strong. This makes the use of refractive lenses practically impossible. Instead, soft x-ray optics have to be based either on reflection or diffraction.

\subsubsection{Multilayer mirrors}

Normal-incidence reflection of soft x-rays on a single interface between two materials cannot be made sufficiently strong for practical applications. This is given by the Fresnel equations (Eqs. 2.5 and 2.6) and was discussed in Chapter 2 The reflectivity can, however, be increased by utilizing the reflections from many interfaces in combination with constructive interference. This is the basic principle of multilayer mirrors.

Multilayer mirrors typically use two materials, which are deposited on a substrate in periodic layers [50]. A third layer can also be used as a barrier between the two primary materials to reduce diffusion. Constructive interference of the individual reflections is achieved if the structure satisfies Bragg's law 


$$
m \lambda=2 d \sin \theta,
$$

where $d$ is the thickness of one bilayer, $\theta$ is the incidence angle (from the surface plane), and $m=1,2,3 \ldots$ is the Bragg order number. In order to optimize the efficiency by minimizing absorption, $m=1$ is preferred. In the case of normal incidence and water-window wavelengths, this gives a bilayer thickness on the order of $1 \mathrm{~nm}$, corresponding to only a few atoms.

The choice of materials is, of course, crucial. To start with, the reflection in each interface should be maximized. Using the Fresnel equations (Eqs. 2.5 and 2.6p at normal incidence, and assuming that $\delta$ and $\beta$ are small, the reflectivity can be written

$$
R=\left|\frac{n_{1}-n_{2}}{n_{1}+n_{2}}\right|^{2} \approx \frac{\Delta \delta^{2}+\Delta \beta^{2}}{4},
$$

where $\Delta \delta$ and $\Delta \beta$ are the differences in the real and imaginary part of the refractive indices. Furthermore, materials with low values of $\beta$ should be chosen in order to reduce absorption.

The number of bilayers $N$ not only determines the total reflectivity, it will also define the spectral resolving power 28,50,

$$
\frac{\Delta \lambda}{\lambda}=\frac{1}{m N} .
$$

The ability of the multilayer mirror to act as a monochromator can be very useful in soft x-ray microscopy. A normal-incidence multilayer condenser in combination with a zone-plate objective can eliminate the need for further filtering of the illumination from the source.

Layer deposition technologies have greatly improved in the last couple of decades and the achieved reflectivity has consequently increased. Some examples of reflectivities at normal or near-normal incidence are $\mathrm{CrN} / \mathrm{B}_{4} \mathrm{C} / \mathrm{Sc}$ $(R=18 \%, \lambda=3.12 \mathrm{~nm})[98, \mathrm{Cr} / \mathrm{Ti}(R=17 \%, \lambda=2.73 \mathrm{~nm})[99, \mathrm{Cr} / \mathrm{V}$ $(R=18 \%, \lambda=2.42 \mathrm{~nm})$ [100, and $\mathrm{Cr} / \mathrm{V}(R=5.6 \%, \lambda=2.48 \mathrm{~nm}) 100$. It should be noted that these numbers were typically presented for small, flat surfaces. Fabrication of large non-flat multilayer mirrors is more challenging. The Stockholm laboratory x-ray microscope (cf. Chapter 4) operates with a state-of-the-art $58 \mathrm{~mm}$ diameter, $350 \mathrm{~mm}$ radius of curvature, spherical condenser mirror, giving $R \approx 4.6 \%$ at $\lambda=2.48 \mathrm{~nm}$ (Paper B).

\subsubsection{Grazing-incidence mirrors}

Another way to increase soft $\mathrm{x}$-ray reflection is to use grazing-incidence angles and the concept of total external reflection. Since $\delta>0$, x-rays will generally refract away from the surface normal, when incident on a more 

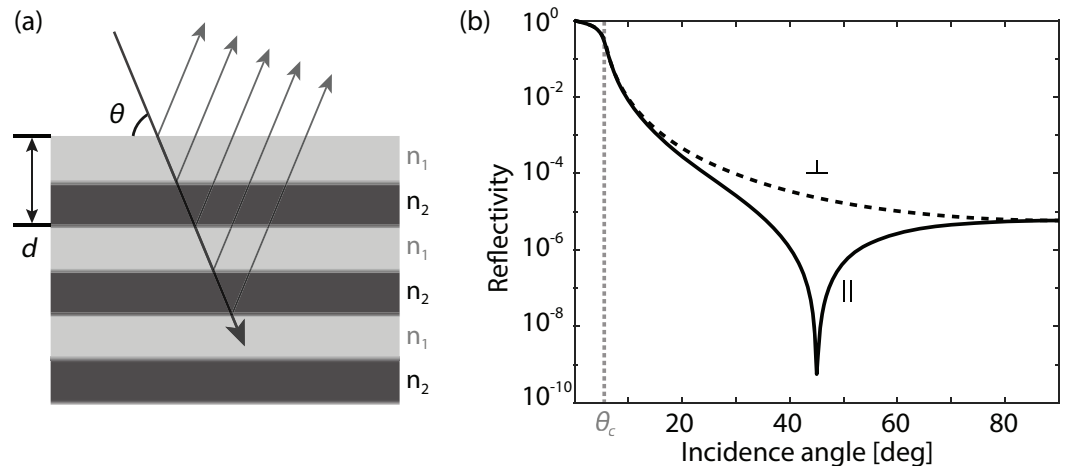

Figure 3.4. Soft x-ray reflective optics are either based on multilayer technology or total external reflection at grazing-incidence angles. (a) Reflections in a multilayer structure with bilayer thickness $d$ and refractive index $n_{1}$ and $n_{2}$ in the two materials. (b) Reflectivity in a nickel surface as a function of incidence angle (from the surface plane), for parallel $\|$ and perpendicular $\perp$ polarized $\lambda=2.48 \mathrm{~nm}$ x-rays.

optically dense material. At incidence angels smaller than the critical angle, given by 27

$$
\theta_{C}=\sqrt{2 \delta}
$$

most of the incident energy will be reflected and total external reflection is achieved. This angle, which depends on material and incident wavelength, sets a limit to the possible x-ray collection geometry. As an example, $\lambda=$ $2.48 \mathrm{~nm}$ x-rays incident on a nickel surface give $\theta_{C}=5.6^{\circ}$. Furthermore, the efficiency of the grazing-incidence mirror, within the critical angle, depends on the surface roughness. Just as for multilayer mirrors, the challenge lies in manufacturing large non-flat optical components, with sufficiently smooth surface.

Grazing-incidence technology is utilized in Kirkpatrick-Baez (KB) mirror pairs 101], ellipsoidal and parabolic glass capillaries 102], or Wolter mirrors [103], for focusing x-rays. Wolter mirrors, which use two reflections on a parabolic and hyperbolic surface, respectively, have the advantage of reducing off-axis aberrations. However, their use is limited by major fabrication challenges 36 .

Grazing-incidence condenser optics are currently used in soft x-ray microscopes, both at synchrotron facilities 104, 105] and in laboratory setups 72,77,89,106]. Some of the laboratory setups also use these types of optics as objectives, although with poor resolution 77,89$]$. 


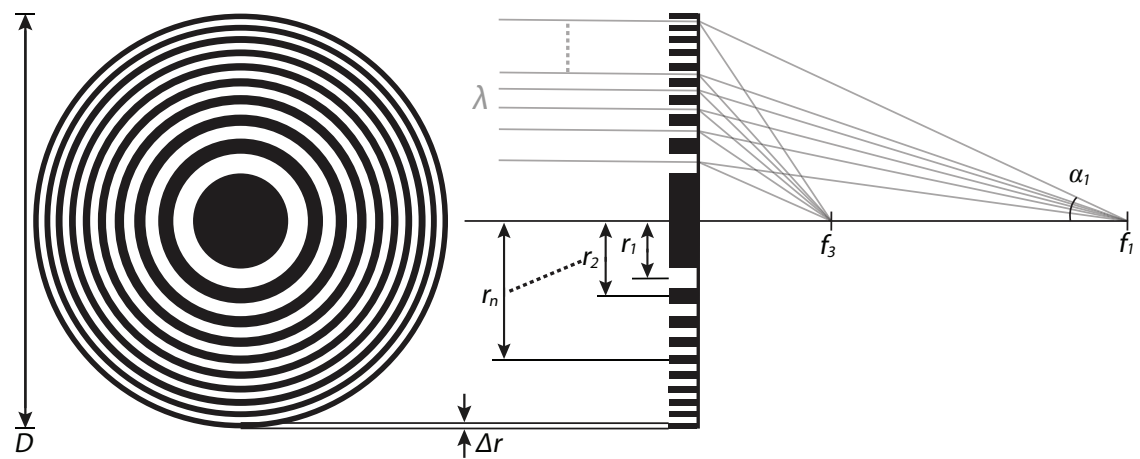

Figure 3.5. The working principle of a Fresnel zone plate. An incident wave front of wavelength $\lambda$ is diffracted more further away from the center, thus causing the waves from all transparent zones to converge at certain points on the optical axis. Only the $1^{\text {st }}$ and $3^{\text {rd }}$ diffraction orders are included in this illustration.

\subsubsection{Zone plates}

The Fresnel zone plate lens, here referred to as a zone plate, utilizes diffraction and constructive interference from a circular grating pattern in order to focus x-rays. The grating period is designed to decrease gradually in the outward radial direction, so that the incident wave front diffracts more the further from the center it is. This way the diffracted waves from all parts of the zone plate can be made to interfere constructively in the same points along the optical axis [50].

Zone plates are extensively used as x-ray focusing optics for a range of applications. Their high resolving power and relatively easy alignment currently make them the number one choice of objective in TXM, and focusing optic in STXM. Furthermore, larger lower-resolution zone plates can be used as condensers in TXM 32 .

Figure 3.5 illustrates the operating principle of a binary zone plate, i.e., with alternating opaque and transparent circles. An incident monochromatic plane wave, here illustrated using horizontal rays, illuminate the entire zone plate and diffracted waves exit the respective transparent zones. A simple geometrical consideration shows that the path difference from adjacent zones to a position $f$ on the optical axis is equal to one wavelength $\lambda$, if the radius $r_{n}$ of each circle (transparent and opaque) is given by

$$
r_{n}^{2}=n \lambda f+\frac{n^{2} \lambda^{2}}{4}
$$

This can be approximated by $r_{n}=\sqrt{n \lambda f}$, by assuming that $f \gg n \lambda / 2$. This is the case for small numerical apertures, defined by $N A=r_{N} / f$, where $N$ 
is the number of the outermost zone.

By defining the outermost zone width $\Delta r=r_{N}-r_{N-1}$, expressions for the diameter $D$, the focal length $f$, and the numerical aperture can be derived,

$$
\begin{aligned}
D & =4 N \Delta r \\
f & =\frac{4 N(\Delta r)^{2}}{\lambda}=\frac{D \Delta r}{\lambda}, \\
N A & =\sin \alpha_{1} \approx \frac{\lambda}{2 \Delta r} .
\end{aligned}
$$

Note that two of the zone-plate parameters, e.g., $D$ and $\Delta r$ or $N A$ and $f$, are sufficient to define the zone-plate pattern for a certain wavelength.

Zone plates also produce higher diffraction orders, given by path differences of multiple half wavelengths $\frac{m \lambda}{2}$, where $m=0, \pm 1, \pm 2 \ldots$ The corresponding focal lengths are given by

$$
f_{m}=\frac{f}{m} .
$$

The negative diffraction orders give negative focal lengths, i.e., virtual foci, and the $m=0$ order corresponds to a wave continuing in its original direction, unaffected by the zone plate. Furthermore, the even orders $m= \pm 2, \pm 4, \pm 6, \ldots$ turn out to cancel for a binary zone plate [50]. The diffraction efficiency in the general case, for a perfect binary zone plate, is given by

$$
\begin{cases}0.25, & m=0 \\ 1 /(m \pi)^{2}, & m \text { odd } \\ 0, & m \text { even }\end{cases}
$$

giving about $10 \%$ in the first diffraction order.

It is possible to improve the zone-plate efficiency by changing the opaque structures for a material that produces a $\lambda / 2$ phase shift [50]. A factor four increase is in theory possible, if the absorption is negligible. Unfortunately, no such material exists. For soft x-rays, a partial increase in efficiency is possible by using nickel as zone-plate material [107]. For hard x-rays gold is the more common choice 108 .

\section{Resolution}

The resolving power of an ideal zone plate can be derived by using the same treatment as for an ideal lens (cf. Chapter 2). Thereby, the highest attainable resolution depends, to a certain degree, on the spatial coherence of the 
illumination. To keep the discussions simple, it is common to use the classical Rayleigh resolution criterion for incoherent point sources (Eq. 2.16). For an ideal zone plate with outermost zone width $\Delta r$, this can be written as

$$
r_{\text {Rayl }}=\frac{0.61 \lambda}{N A}=1.22 \Delta r
$$

At this point it is important to interpret what the resolution limit actually means in the real imaging case. For this we consider a grating test sample of period $L$. The case of fully incoherent imaging corresponds to illumination of the grating from all angles. When $L$ approaches the resolution limit, each transparent line in the grating can be seen as an incoherent point source, and will thus be resolved if $L \geq r_{\text {Rayl }}$. Fully coherent imaging would instead correspond to illumination by a normally incident plane wave. In this case, the resolution would almost be cut in half, albeit with better contrast (cf. Fig. 2.3).

The common choice in microscopy (including TXM) is to match the numerical aperture of the condenser to the numerical aperture of the objective. Even though the illumination angle will be somewhat limited, this case can with fairly good accuracy be approximated as incoherent imaging. The resolution limit given by Eq. 3.17 is therefore typically referred to as the full-period resolution in TXM. More detailed discussions on the resolution for partially coherent illumination can be found in 48,50, 109 .

\section{Depth of focus and spectral bandwidth}

Due to the diffraction-limited focus spot, some deviation from the ideal focus is acceptable. This is usually described by the depth of focus (DOF), defined by the (longitudinal) distance from the focus where the intensity of the spot is decreased by $20 \%$. For a zone plate in the $1^{\text {st }}$ order focus, this is given by

$$
\Delta z= \pm \frac{\lambda}{2(N A)^{2}}=\frac{2(\Delta r)^{2}}{\lambda} .
$$

Zone plates are chromatic by nature, meaning that the focal length (Eq. 3.13 depends on the wavelength. However, by a similar argument as for the DOF, a certain spectral bandwidth is acceptable. As a rule of thumb, chromatic aberrations can be avoided if the spectral bandwidth of the illumination satisfies

$$
\frac{\Delta \lambda}{\lambda} \leq \frac{1}{N}
$$


In this case the focal shift due to the spectral bandwidth does not exceed the DOF. Here it should be noted that Eq. 3.19 only describes the size of the chromatic aberrations relative to the diffraction spot. For example, if the outermost zone width $\Delta r$ is increased, which results in a larger focus spot (cf. Eq. 3.17), the chromatic aberrations also increase, even if $N$ is kept constant. The absolute size of the blur due to chromatic aberrations is proportional to the zone-plate diameter $D$.

\subsection{Optical arrangements for TXM}

Designing an arrangement for TXM basically means deciding on a source, a condenser and an objective. The other parts of the system will, to a large extent, follow from these decisions. It should be kept in mind that all components of the microscope need to be kept in vacuum, or in some cases a helium atmosphere, due to the absorption properties of soft x-rays (cf. Chapter 2). Figure 3.6 depicts four possible condenser-objective combinations for the case of a polychromatic source, radiating in all directions. Since the efficiency of soft x-ray optics is low, the condenser and the objective typically cannot consist of more than one part, especially if the source brightness is limited. This prevents implementation of the otherwise preferable Köhler illumination, which is used in full-field visible-light microscopy.

An arrangement consisting of a zone-plate condenser in combination with a zone-plate objective is shown in Fig. 3.6.(a). In this case the x-rays from the source have to be monochromatized, e.g., by using metal filters with sharp absorption edges. Furthermore, a central stop (CS) is needed to prevent the $0^{\text {th }}$ diffraction order from reaching the detector, and an order sorting aperture blocks the higher diffraction orders from the condenser.

Figure 3.6.(b) shows an achromatic arrangement, in which grazing-incidence reflective optics are used both as condenser and objective. This has the advantage of transmitting more x-rays through the system. However, drawbacks include difficulties in alignment and challenges in fabricating a high-resolution objective. This arrangement needs a CS to block direct illumination from the source, but no monochromator.

The combination of a grazing-incidence-mirror condenser and a zoneplate objective is shown in Fig. 3.6.(c). Here, the polychromatic radiation from the condenser has to be monochromatized before reaching the zoneplate objective. Finally, Fig. 3.6(d) shows a normal-incidence multilayermirror condenser in combination with a zone-plate objective. A major advantage of this arrangement is that the condenser acts as monochromator, thus eliminating the need for further spectral filtering. Furthermore, the 
(a)

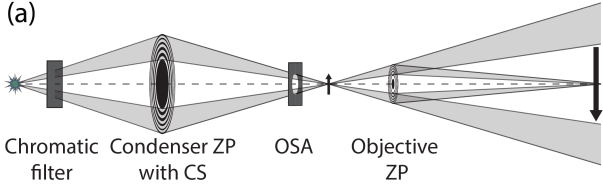

with CS

(c)

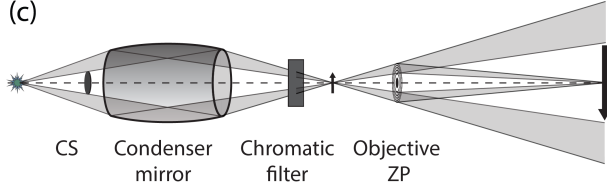

(b)

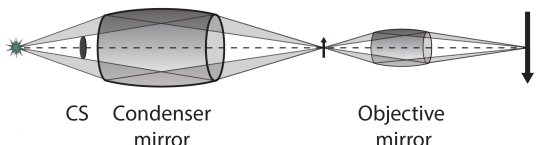

(d)

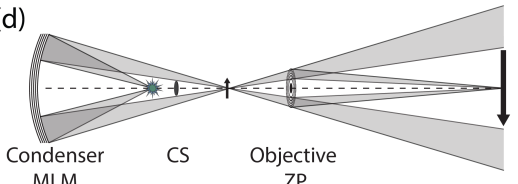

Figure 3.6. Optical arrangements for TXM with an extended polychromatic source. (a) The combination of a zone-plate (ZP) condenser and an objective zone plate requires a chromatic filter, a central stop (CS) and an order sorting aperture (OSA). (b) An achromatic arrangement, using grazing incidence optics as condenser and objective, only requires a central stop. (c) The combination of grazing incidence condenser optics and a zone-plate objective still requires a chromatic filter, preferably placed before the sample. (d) A normal-incidence multilayer condenser mirror (MLM) acts as a monochromator and can thus be combined with a zone-plate objective, without additional chromatic filters.

alignment of a normal-incidence multilayer-mirror condenser is easy, compared to the other alternatives.

All arrangements depicted in Fig. 3.6 have been demonstrated in TXM, at synchrotron-radiation facilities or in laboratory setups. Some examples are (a) [110, (b) 77, 89, (c) 72, 104 106], and (d) 80,111.

\subsection{Sample environment}

Sample handling and preparation is crucial for a successful imaging experiment. Compared to other techniques, like TEM or super-resolution light microscopy, the sample preparation for x-ray microscopy is simple, which is often recognized as one of its major advantages. The natural contrast and the appropriate penetration capability of the x-rays eliminate the need for both sectioning and staining.

The samples for soft x-ray microscopy are typically placed on few mmsized TEM grids, consisting of a thin carbon layer on a gold or copper grid structure. The grid is then mounted on a specialized sample holder that can be inserted into the vacuum of the microscope. The sample holder should also allow fine positioning of the sample (this can also be done by moving the objective) as well as rotation for tomography.

An alternative to the TEM grids is provided by thin-walled glass capillaries 112,113. These have the advantage of allowing a full $180^{\circ}$ tilt for tomography, thereby eliminating the missing-wedge problem. Disadvan- 
tages include a higher x-ray attenuation in the capillary walls, compared to the holey-carbon layer of the TEM grids, which is typically a problem for laboratory-based microscopes. Furthermore, the capillaries are unsuitable for samples that require a surface, like adherent cells and cell-cell interactions.

\subsubsection{Cryofixation}

The standard sample-preparation method for biological samples is cryofixation, where the sample is quickly cooled to cryogenic temperatures $\left(<-150{ }^{\circ} \mathrm{C}\right)$ and kept there during imaging. By doing this, the sample will be spatially fixed, which is necessary since the exposure times are typically on the order of seconds or tens of seconds. Furthermore, the vitreous (amorphous) ice structure, formed during the rapid cooling, will prevent radiation damage while still maintaining the morphology of the sample. Studies have shown that up to $10^{10}$ Gy can be delivered to cryofixed cell samples with no visible alterations to the morphology [114], while $10^{4}$ Gy is enough to damage non-frozen cells [115. Achieving high-resolution images with a sufficient SNR typically requires doses on the order of $10^{5}-10^{8}$ Gy 116 .

Cryofixation for soft x-ray microscopy is usually done by plunge freezing the sample in a liquid cryogen with appropriate cooling properties. Ethane is a good choice due to the large gap between its melting and boiling temperature $(90-185 \mathrm{~K})$, which prevents an insulating gas layer from forming around the sample 117. A simple plunge-freezing setup with liquid ethane can form vitreous ice through samples up to $\sim 10 \mu \mathrm{m}$ thick 116.

High-pressure freezing is an efficient but more complicated alternative to plunge freezing. Here, a pressure of 2000 bar is applied within a few milliseconds, before a flow of liquid nitrogen cools the sample to cryogenic temperatures. This way, up to $200 \mu \mathrm{m}$ thick objects can be frozen without forming crystalline ice. The drawbacks of high-pressure freezing are the high cost of the machine and the challenge in controlling the ice thickness, which can be crucial in biological soft x-ray microscopy.

\subsubsection{Chemical fixation}

Chemical fixation methods, such as glutaraldehyde fixation, have been applied in biological x-ray microscopy 118. However, studies show severe morphological alterations to the samples, as well as radiation-induced damage, thus making these methods inappropriate for most applications 119 . 120 . 


\subsubsection{Wet imaging}

Wet imaging is also possible in cases where radiation damage is not an issue. As an example, environmental colloids in aqueous suspension have been studied using the Stockholm laboratory x-ray microscope [121]. Here, the samples were clamped between two $\mathrm{Si}_{3} \mathrm{~N}_{4}$-membranes and kept in a helium atmosphere, separated from the vacuum of the microscope.

\subsection{X-ray detection}

The two possible techniques for soft x-ray detection in TXM are the chargecoupled device (CCD) and the complementary metal-oxide-semiconductor (CMOS) sensor. While CMOS can provide faster read-out, the CCD typically has lower noise and higher dynamic range, and is therefore the most common choice. Commercially available scientific-grade CCDs currently provide a quantum efficiency of up to $85 \%$ at $500 \mathrm{eV}$ photon energy, with a negligible dark current $\left(\right.$ at $\left.<-50{ }^{\circ} \mathrm{C}\right)$ and a readout noise on the order of 10 electrons/pixel [122]. This should be compared to the $>100$ electrons generated for each detected $500 \mathrm{eV}$ x-ray. The high quantum efficiency is achieved by a back-illuminated arrangement, where the read-out electronics are placed on the backside of the sensitive CCD chip to avoid blocking any incident radiation.

When designing an arrangement for TXM, the effective pixel size in the sample plane must be taken into consideration in order not to limit the resolution. The actual pixel size, divided by the magnification, should not exceed half the smallest resolvable period $L$ (cf. discussion in Section 3.3.3). This is the well-known Nyquist sampling criterion, which can be written as

$$
r_{p i x} \leq \frac{L}{2} .
$$

In practice, however, the pixel size should be at least four times smaller than the resolvable period for the decrease in contrast to be negligible.

In STXM there is no need for spatial resolution at the detector, and thus, photomultiplier (PMT) systems are typically used due to their extreme sensitivity and detection speed 123 . 



\section{Chapter 4}

\section{The Stockholm laboratory x-ray microscope}

With an early version of the Stockholm laboratory x-ray microscope, Hertz et al. demonstrated the first laboratory-scale sub-visible-resolution soft xray microscopy 80 . Since then, the system has undergone major improvements with regard to x-ray flux, sample environment, stability and general reliability. This Chapter describes the general arrangement of the Stockholm laboratory x-ray microscope, as well as details about its main components and operational principle. Focus will be on the technical developments that enabled the first comprehensive biological studies with high scientific relevance. The biological applications are further discussed in Chapter 5

\subsection{Microscope arrangement}

The Stockholm laboratory x-ray microscope arrangement is based on the combination of a normal-incidence multilayer condenser mirror and a highresolution zone-plate objective (cf. Fig 3.6 (d)). The x-ray source is a liquidnitrogen-jet laser plasma, which emits a combination of bremsstrahlung and strong line emission. As discussed in Section 3.4 the multilayer condenser works as a spectral filter, creating the monochromatic illumination needed by the zone plate. A thin $(100-200 \mathrm{~nm})$ aluminum filter is also needed to block stray light from the laser. Furthermore, a central stop, placed between the source and the sample, blocks direct illumination from the source and creates the hollow cone needed to keep the $0^{\text {th }}$ order radiation away from the detector. In addition to the main components discussed in this Chapter, apertures and shutters are placed in a few positions along the optical axis to block stray radiation and protect sensitive optics. A schematic illustration 


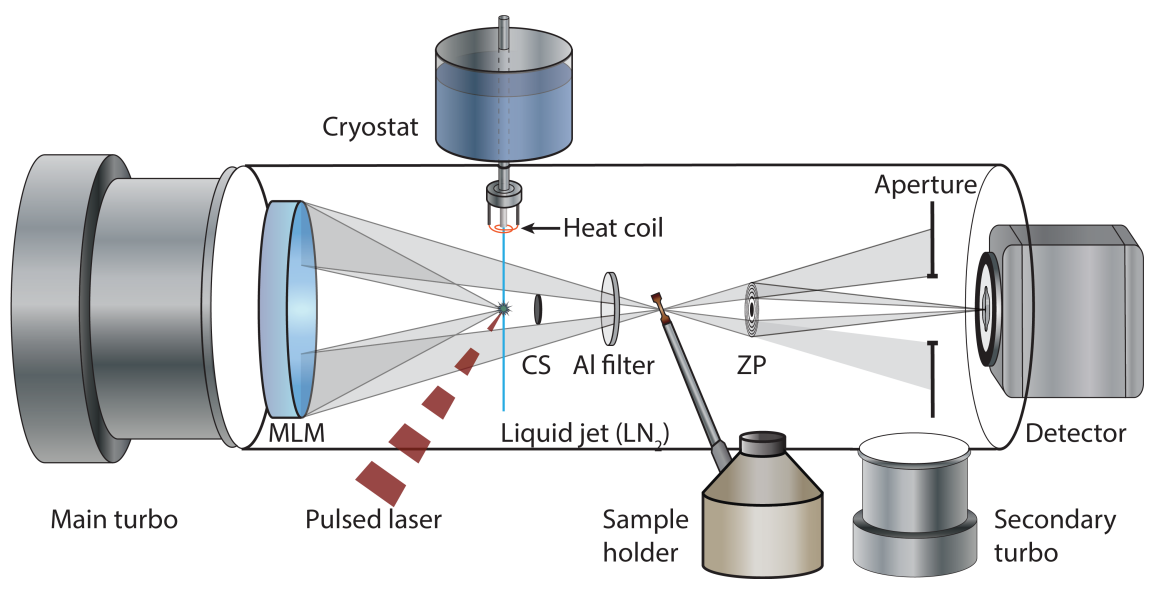

Figure 4.1. The Stockholm laboratory x-ray microscope arrangement, including source components, optics, sample holder, detector and vacuum system. $\mathrm{MLM}=$ multilayer mirror, $\mathrm{LN}_{2}=$ liquid nitrogen, $\mathrm{CS}=$ central stop, $\mathrm{Al}=$ aluminum, $\mathrm{ZP}=$ zone plate.

of the Stockholm laboratory x-ray microscope arrangement, as described in this Chapter, is shown in Fig. 4.1

\subsection{Source}

The liquid-nitrogen-jet laser-plasma x-ray source provides high spectral brightness in the water window, due to strong line emission at $\lambda=2.48 \mathrm{~nm}$ $(500 \mathrm{eV})$ and $\lambda=2.88 \mathrm{~nm}(431 \mathrm{eV})$. The corresponding electron transitions, from the $n=2$ state to the $n=1$ state, are possible in the fully or almost fully ionized nitrogen atoms, at plasma temperatures around $10^{6} \mathrm{~K}$. The $\lambda=2.48 \mathrm{~nm}$ and $\lambda=2.88 \mathrm{~nm}$ lines are often referred to as hydrogenlike and helium-like, respectively, referring to the number of electrons lefts in the ions 71 . The Stockholm laboratory x-ray microscope utilizes the hydrogen-like $\lambda=2.48 \mathrm{~nm}$ line, due to its position in the higher-energy part of the water window (cf. Fig. 2.2). The bandwidth of this line was measured during the early development of the liquid-nitrogen laser-plasma source, giving $\Delta \lambda / \lambda \leq 1 / 500$ 124.

The nitrogen plasma is created by focusing a high-power $\lambda=1064 \mathrm{~nm}$ laser, with 600 ps pulses and a repetition rate of $2 \mathrm{kHz}$ (Fraunhofer ILT, Aachen) [125], onto a liquid-nitrogen jet in vacuum. The diode-pumped, slab-amplified laser can provide an average power of $>200 \mathrm{~W}$, resulting in an x-ray brightness that approximately scales linearly with the power 83. In 2012 Martz et al. demonstrated a brightness of 


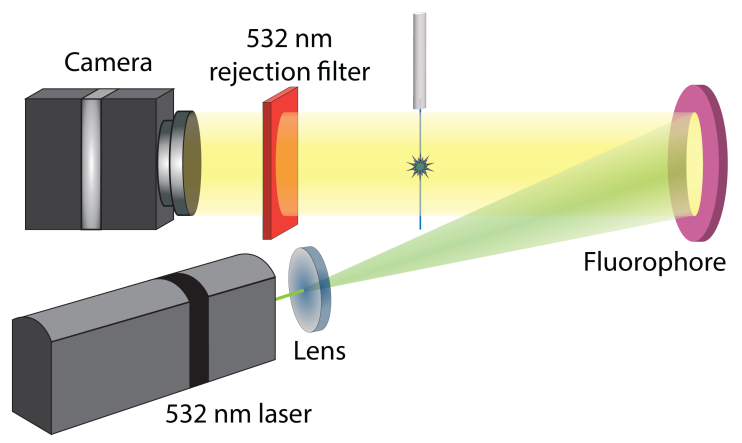

Figure 4.2. Setup for real-time flash-imaging of the liquid-nitrogen jet.

$>10^{12}$ photons $/\left(\mathrm{s} \times \mathrm{sr} \times \mathrm{mm}^{2} \times 0.1 \% \mathrm{BW}\right)$ at $200 \mathrm{~W}$. However, the source is typically operated at more moderate laser powers $(<100 \mathrm{~W})$ in order to maintain the stability for long-term operation. Measurements have shown that the size of the source is typically $14 \times 20 \mathrm{\mu m}^{2}$ full width at half maximum (FWHM).

The liquid jet is formed by driving high-purity $(99.99999 \%)$ gaseous nitrogen, at typically 8-15 bar, through a custom-built liquid-nitrogen-cooled cryostat. By pumping on the cryostat, its temperature is regulated to achieve an adequate cooling rate. The liquefied high-purity nitrogen is finally ejected into the microscope vacuum chamber through a $30 \mu \mathrm{m}$ diameter glass capillary nozzle.

\subsubsection{Source stability}

The liquid-jet stability is of crucial importance for the high-brightness and high-stability operation of the laser-plasma source. This was investigated in great detail in Paper A. During operation, the jet is monitored by a flash-imaging system, consisting of a $4 \mathrm{~ns}$ green $(\lambda=532 \mathrm{~nm})$ laser, a liquid fluorophore, a $532 \mathrm{~nm}$ rejection filter and a camera that is triggered by the laser pulses. This arrangement is shown in Fig. 4.2 With the realtime feedback provided by the flash-imaging system, the driving pressure is regulated to achieve optimal jet stability.

One of the main results of Paper A was the implementation of a radiative heating element, mounted close to the tip of the nozzle. The heating element, consisting of a highly resistive wire coiled around the nozzle, is included in Fig. 4.1. It was shown that the liquid nitrogen, when ejected into vacuum, will freeze close to the nozzle and possibly cause asymmetric disturbances. By applying heat close to the nozzle tip, the freezing is delayed and the jet stability is improved. Adjusting the current through 
the resistive heating element gives additional control to the behavior of the liquid-nitrogen jet.

Furthermore, preventive measures have to be taken to avoid clogs in the nozzle. A new procedure was developed to clean the nozzle prior to operation. This includes flushing methanol and subsequent heated nitrogen gas through the cryostat, and monitoring the process in a light microscope. Fine filters are also used to prevent particles from reaching the nozzle through the cryostat.

Finally, the quality of the laser beam is closely monitored for optimal performance, both prior to and during microscope operation. For this purpose, an $\mathrm{M}^{2}$ measurement system is permanently mounted on the optical table next to the x-ray microscope. Any sensitive optics along the beam path, especially the entrance window to the vacuum chamber, are also checked and changed when needed.

\subsection{Condenser mirror}

Together with the improved source stability, the implementation of a new multilayer condenser mirror has made the highest impact, in the last several years, on the overall performance of the Stockholm laboratory x-ray microscope. The new mirror (OptiX fab, Jena) 126 consists of $500 \mathrm{Cr} / \mathrm{V}$ double layers with $\mathrm{B}_{4} \mathrm{C}$ barriers to decrease material interdiffusion. The average reflectivity for $\lambda=2.48 \mathrm{~nm}$ at normal incidence is $>4.5 \%$ over the used mirror area. This can be compared with a reflectivity of $0.6 \%$ for the previous condenser mirrors used in the Stockholm laboratory x-ray microscope. Figure 4.3 shows the result of a reflectivity measurement over the entire condenser-mirror area.

The condenser mirror is spherical with a radius of curvature of $350 \mathrm{~mm}$ and a diameter of $58 \mathrm{~mm}$. It is positioned $285 \mathrm{~mm}$ behind the source, giving an $M=1.6$ magnified image of the source in the sample plane. With an actual source size of $14 \times 20 \mathrm{\mu m}^{2}$, this gives an illuminated area of $22 \times 32 \mu^{2}$ FWHM. However, it should be noted that the source typically moves somewhat during the exposures, due to a combination of jet and laser instabilities, resulting in a slightly larger effective illuminated area.

The common choice in microscopy is to match the numerical aperture of the condenser to the numerical aperture of the objective. This gives a resolution corresponding to the Rayleigh criterion for incoherent point sources (cf. Section 3.3.3. However, the theory of partially coherent imaging finds that the resolution can be somewhat increased by making the numerical aperture of the condenser slightly $(\sim 1.5 \times)$ larger [48]. The current condenser arrangement results in an illumination angle of $0.063 \mathrm{rad}$, corresponding to the numerical aperture of a $\Delta r=20 \mathrm{~nm}$ zone plate at $\lambda=2.48 \mathrm{~nm}$. The 


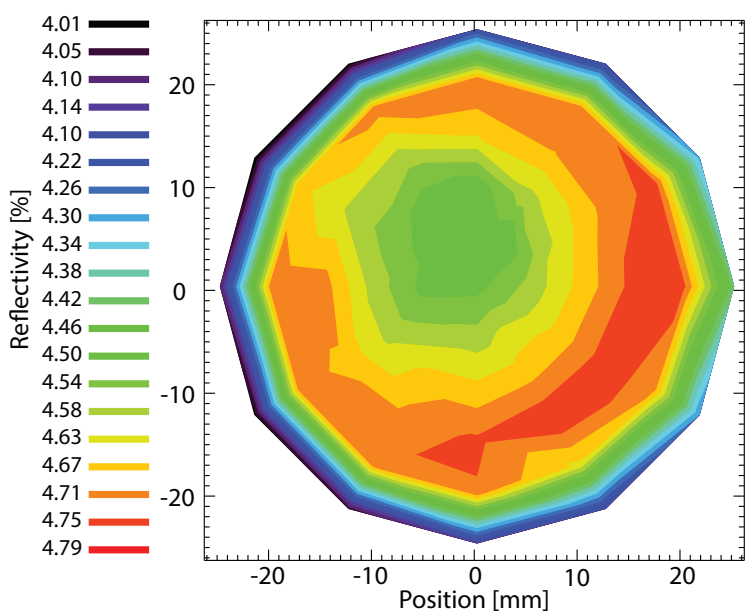

Figure 4.3. Reflectivity measurement of the multilayer condenser mirror. Data from 126 .

$\sim 1.5 \times$ overmatching can be achieved by choosing a zone-plate objective with $\Delta r=30 \mathrm{~nm}(N A=0.0413)$.

\subsection{Objective zone plate}

As described in Chapter 3 the objective zone plate determines several important imaging parameters and should ideally be customized for each imaging application. Yet, choosing the optimal zone plate is not trivial, since the physical and optical zone-plate parameters affect each other in intricate ways (cf. Eqs. 3.12 3.19). As an example, the outermost zone width $\Delta r$ sets the resolution limit due to diffraction, and should therefore be made small. However, $\Delta r$ also affects the focal length $f$ (which should not be made too small), the numerical aperture $N A$ (which should match the condenser), the DOF (which should be adjusted depending on the sample thickness) and the number of zones $N$ (which, if made loo large, may lead to chromatic aberrations, depending on the bandwidth of the source). It is obvious that the choice of zone-plate parameters must be based on a balanced compromise, determined by the imaging task at hand.

The objectives used in the Stockholm laboratory x-ray microscope are typically nickel zone plates with diameters ranging from 50 to $200 \mu \mathrm{m}$, and outermost zone widths between 30 and $40 \mathrm{~nm}$. In Paper B the goal was to perform tomography of whole cells and cell-cell interactions, and in order to facilitate risk-free rotation of the sample, a zone plate with a large focal 
length was chosen. The zone-plate parameters used were $\Delta r=30 \mathrm{~nm}$ and $D=200 \mu \mathrm{m}$, giving $f=2.4 \mathrm{~mm}$. It was noted that the large number of zones $(N \approx 1660)$ was likely to cause some chromatic aberrations, due to the finite source bandwidth. In Paper C, a $\Delta r=30 \mathrm{~nm}, D=100 \mu \mathrm{m}$ zone plate was used in order to achieve high resolution in a flat test sample. An even smaller outermost zone width could have been used here, if one had been available.

\subsection{Sample stage}

The sample stage is a modified TEM goniometer stage (FEI) that enables translation in all three dimensions as well as tilt along one axis. During operation the sample is typically moved in the plane perpendicular to the optical axis, over areas of up to $2 \times 2 \mathrm{~mm}^{2}$. Scanning through large sample areas with short exposures is often necessary in order to find regions of certain interest, where longer-exposure images are acquired.

The Stockholm laboratory x-ray microscope allows imaging of cryofixed samples, by using a temperature-controlled cryo sample holder. After cryofixation by plunge freezing (cf. Section 3.5), the $3 \mathrm{~mm}$ sample grids are kept in liquid nitrogen and then mounted on the sample holder in a specialized cryogenic stage. The sample holder is then inserted into the microscope through a vacuum sluice, and a temperature-control unit is connected. During imaging, the sample is kept at constant temperature of typically $-165 \pm 0.01^{\circ} \mathrm{C}$.

Tomographic data sets can be acquired by utilizing the tilt along the allowed axis. The stage allows angular steps of $<0.1^{\circ}$, even though $1^{\circ}$ steps are enough for the current system. Unfortunately, the current microscope arrangement does not allow exact alignment of the zone-plate focus with the rotation axis of the sample stage. The sample will therefore move somewhat during rotation, both in the image plane and along the optical axis. Thus, continuous repositioning and refocusing are required during tomographic data acquisition. Possible ways to improve this procedure include the implementation of automatic repositioning and refocusing during rotation, or an accurate pre-alignment of the zone-plate focus with the axis of rotation. The latter has already been implemented at a similar microscope setup in Berlin, and is described in Paper F.

\subsection{Detector}

The detector is an air-cooled back-illuminated CCD (Andor) with $2048 \times$ 2048 pixels and a pixel size of $13.5 \mu \mathrm{m}$. The dark current and readout noise are kept negligible at temperatures $<-50{ }^{\circ} \mathrm{C}$ and readout speeds of up to 
$3 \mathrm{MHz}$ (giving $1.6 \mathrm{~s}$ per frame). During initial scanning of the sample, a $4 \times 4$ pixel binning is used to speed up the readout time to $<0.5 \mathrm{~s}$.

The microscope magnification can be adjusted by sliding the detector along the optical axis. Typical values of $M=700-1200$ are used, which give effective pixel sizes of 11-19 $\mathrm{nm}$ in the sample plane. As mentioned in Section 3.6 the pixel size should ideally not exceed a quarter of the fullperiod resolution. In other words, at least double the Nyquist sampling frequency is desired.

\subsection{Vacuum system}

The $\lambda=2.48 \mathrm{~nm}$ x-rays require medium to high vacuum, not to be absorbed on their way through the microscope. With a $10^{-2}$ mbar air atmosphere, about $96 \%$ of the x-rays are transmitted over a distance of $2 \mathrm{~m}$. At $10^{-3}$ mbar the corresponding value is about $99.6 \%$ [28.

The vacuum system of the Stockholm laboratory x-ray microscope consists of three roughing pumps, connected to each end of the microscope and to a small hole where the liquid-nitrogen jet exits the source chamber (called the jet dump). Furthermore, two turbo pumps, one at each end of the microscope, are used to further improve the vacuum. The source chamber can, at any time, be closed off from the rest of the microscope by using an air-operated valve. This in an important feature that helps protecting sensitive parts, like the aluminum filter, the zone plate, and the detector, in case the liquid-nitrogen jet becomes unstable.

Prior to operation, the vacuum in the microscope typically reaches below $10^{-6}$ mbar. When the source is being operated, the pressure increases to about $10^{-3}-10^{-2}$ mbar in the source chamber and about $10^{-5}$ mbar close to the detector.

\subsection{System stability}

High-resolution microscopy sets high demands on the mechanical stability of the instrument. The most sensitive imaging components in the Stockholm laboratory x-ray microscope are the sample and the objective. Movements in these parts during image acquisition result in a blurred image, limiting the resolution to approximately the same magnitude as the movements. The detector could in theory also affect the resolution, but here the sensitivity is on the order of $1000 \times$ smaller, due to the high magnification. The source and condenser, on the other hand, can affect the shape and position of the illuminated area, but not the image resolution [48].

The microscope stability and its effect on the achievable image resolution were investigated in Paper C. A fiber interferometer and real-time fast 

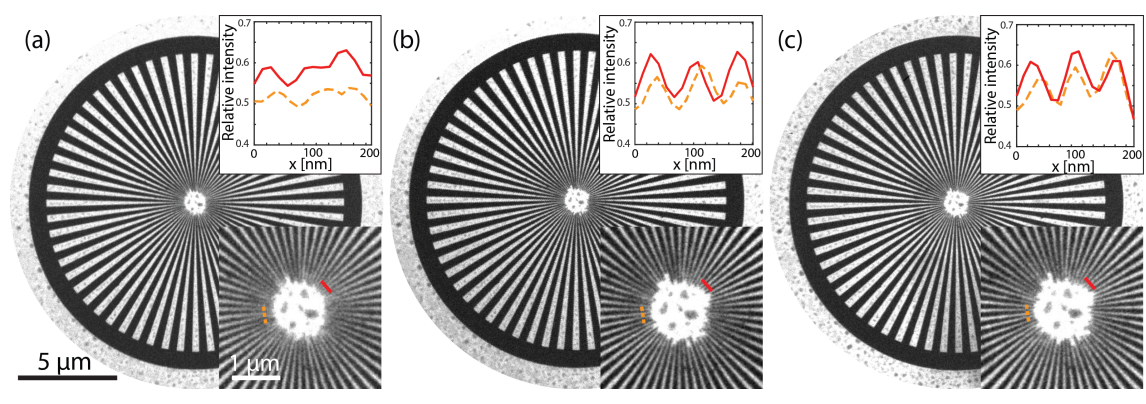

Figure 4.4. Images of a gold Siemens star, illustrating the improved resolution from the stability investigation. (a) Before stability investigation. (b) After the stability investigation. (c) After the stability investigation, with Siemens star cooled to cryogenic temperatures $\left(-165^{\circ} \mathrm{C}\right)$. (Figure adapted from Paper $\left.\mathrm{C}\right)$.

Fourier transform (FFT) analysis were used to measure and characterize the movements of the zone-plate mount and the sample holder. This was done at both short and long time scales, and in vacuum to simulate real operating conditions. The results revealed $\mathrm{kHz}$-range vibrations with an amplitude of up to $30 \mathrm{~nm}$, which is clearly on the same order of magnitude as the resolution. Using the vibration-frequency spectrum obtained from the FFT analysis, the main turbo pump (cf. Fig. 4.1) could be identified as the main source of vibrations. The vibrations could subsequently be reduced by making some modifications to the microscope setup. The resulting improvement in resolution was documented by imaging a gold Siemens star test structure, with lines down to $30 \mathrm{~nm}$ wide. Images before (Fig. 4.4 (a)) and after (Fig. 4.4 (b)) the stability investigation show a significantly decreased blurring of the center lines. This is especially clear when comparing the included profile plots. Images of finer test structures later showed a resolution down to $25 \mathrm{~nm}$ half period.

Furthermore, Paper $\mathrm{C}$ also investigated the stability in the presence of liquid nitrogen in the system (cryostat and cryo sample holder), and both slow drifts and fast vibrations were measured. These instabilities will, however, stay acceptably small as long as the temperatures in the cryostat and cryo sample holder stabilize. Figure. 4.4(c) shows the gold Siemens star, mounted on the cryo sample holder and imaged at $-165{ }^{\circ} \mathrm{C}$. It proves the microscope's ability to produce high-resolution images of samples under cryogenic conditions. 


\section{Chapter 5}

\section{Biological applications}

The technical developments described in Chapter 4 have enabled the first comprehensive biological studies with high scientific relevance, including whole-cell cryotomography with few tens of minutes exposure time. This means that the Stockholm laboratory x-ray microscope can now provide a competitive alternative to synchrotron-based microscopes, even if the image quality is not quite at the same level. The advantages of a laboratory-based system become apparent in studies that require tedious and iterative optimization of sample preparation parameters, or when larger sets of samples are studied over time. Furthermore, the integration with complementary methods is facilitated by the close access to the home laboratory environment. This Chapter will discuss recent biological studies performed using the Stockholm laboratory x-ray microscope. An outlook on future applications is also given.

\subsection{Starvation-induced autophagy in HEK 293T cells}

Paper B presents the first routine cryo-imaging of whole cells with 10-20 s exposure time, using the Stockholm laboratory x-ray microscope. Human embryonic kidney (HEK 293T) cells were used in the study due to their reliable growth and surface adhesion. This cell line is commercially available and has been used in biological research and by the biotechnology industry for many years. The cells were kept in cell culture medium under physiological conditions $\left(37^{\circ} \mathrm{C}\right.$ and $\left.5 \% \mathrm{CO}_{2}\right)$. In order to maintain the culture, new medium was added every one to two days.

The HEK 293T cells were imaged with the Stockholm laboratory xray microscope under different stages of starvation, and changes to the cell morphology were studied. Starvation was induced simply by not adding any new cell medium for up to three days. It was noted that the degree of 


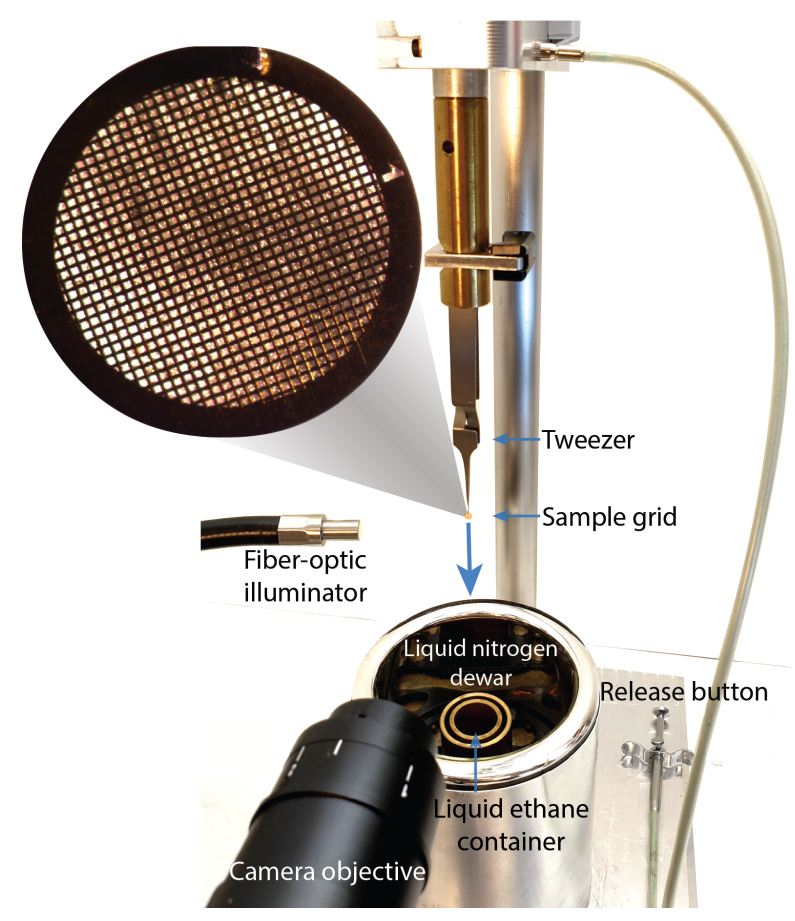

Figure 5.1. Custom-built plunge-freezing setup with camera monitoring and illumination at a grazing angle. The inset shows a blow-up of the sample grid.

starvation was very approximate. However, effects of the starvation could be clearly noticed and documented, and thereby the goal of this demonstrative study was achieved.

The challenges in the sample preparation for this study mainly consisted of two parts. The first was to seed an appropriate concentration of cells onto the gold TEM grids, so that (ideally) cells would adhere to each $\sim 55 \times 55 \mathrm{\mu m}^{2}$ square, while still not overlapping or blocking each other during tomography. The second challenge was to achieve an ice-layer thickness of about 5-10 $\mathrm{mm}$, in order to keep the cells hydrated, while still allowing high x-ray transmission. Both challenges were overcome by incorporating a simple microscope into the plunge-freezing setup (Fig. 5.1). This microscope consisted of a camera, a magnifying objective, and illumination of the grid at a grazing angle. The cells were seeded onto the same side as the gold bars and the grids were blotted from behind to adjust the liquid-layer thickness. After optimizing the angle and intensity of the illumination, a shadow effect clearly showed when the liquid layer reached below the height of the bars $(10 \mu \mathrm{m})$. At the same time, the number of cells on the grid was monitored, 

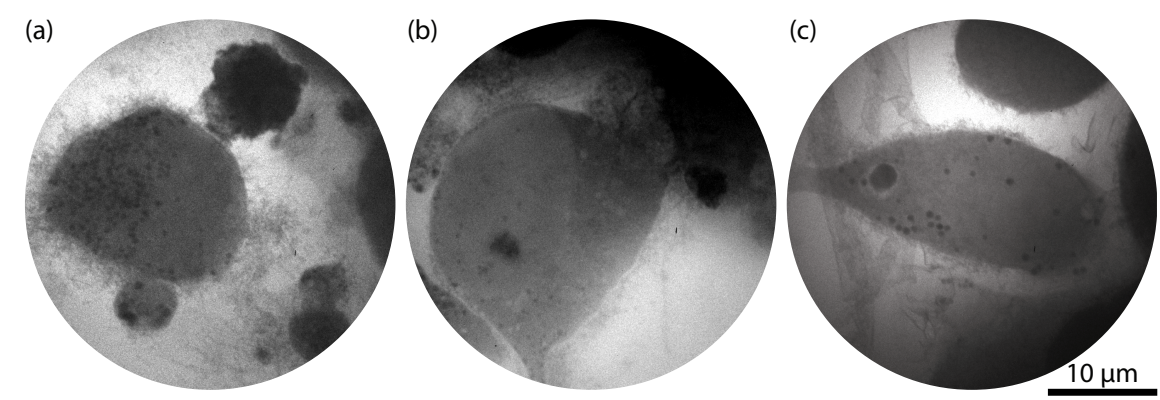

Figure 5.2. Examples of cryofixed HEK $293 \mathrm{~T}$ cells imaged using the Stockholm laboratory x-ray microscope. Exposure times (a) $10 \mathrm{~s}$, (b) $20 \mathrm{~s}$, and (c) $30 \mathrm{~s}$. Scale bar $10 \mu \mathrm{m}$.

which provided valuable feedback for adjusting the cell concentration for subsequent sample preparation.

Figure 5.2 shows three examples of HEK 293T cells imaged using 10-30 s exposures in the Stockholm laboratory x-ray microscope. The effects of the starvation included development of carbon-dense vesicles (typically 0.4-0.9 $\mu \mathrm{m}$ diameter), which could be related to autophagy [127,128]. Furthermore, the starvation would typically result in a rounded cell shape and decreasing adhesion. Further discussion and results, including tomography of a starved HEK 293T cell, are presented in Paper B.

\subsection{NK-cell target-cell interaction}

The advantages of a laboratory-based system can be further appreciated in studies of cell-cell interactions. Here, a large set of samples has to be scanned in order to get an overview of the interaction process. The challenges in sample preparation are extended to finding the appropriate concentration of two cell types, and the suitable timing parameters for mixing the cells. Some feedback can be obtained using a visible-light microscope. However, an iterative optimization of the sample preparation based on the results of $\mathrm{x}$-ray microscopy will provide the necessary information.

In Paper B the interaction between primary IL-2 activated natural killer (NK) cells and HEK 293T target cells was studied. NK cells, which are an important part of the innate immune system, have the ability to identify and kill target cells in a carefully regulated process [129,130]. The NK-cell target-cell interaction can be divided into several distinct steps, starting with recognition and initial adhesion, and ending with the delivery of lytic granules that eventually causes the target cell to lyse. 

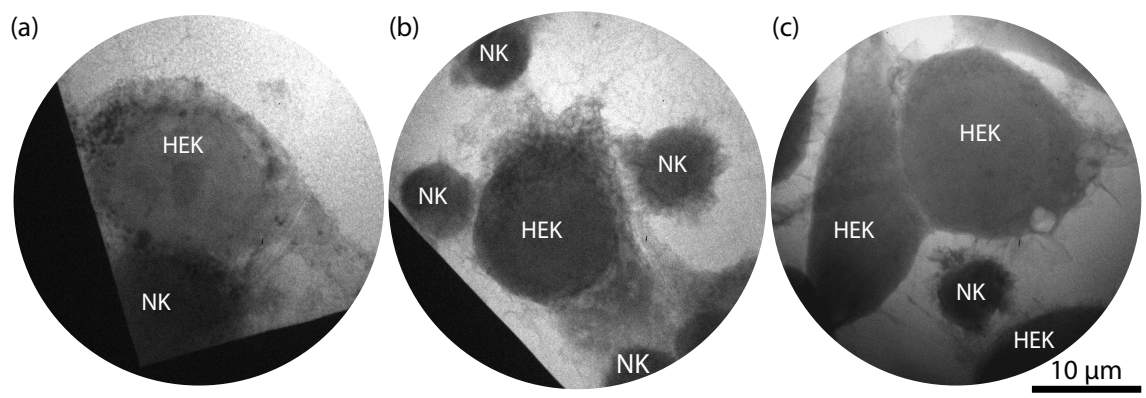

Figure 5.3. The interaction between primary IL-2 activated NK cells and HEK $293 \mathrm{~T}$ target cells, imaged under cryogenic conditions with the Stockholm laboratory x-ray microscope. Exposure times (a) $10 \mathrm{~s}$, (b) $20 \mathrm{~s}$, and (c) $30 \mathrm{~s}$. Scale bar $10 \mu \mathrm{m}$.

Figure 5.3 shows three examples of NK-cell HEK-cell interaction, imaged using 10-30 s exposures in the Stockholm laboratory x-ray microscope. The typically 5-10 $\mathrm{\mu m}$ diameter primary NK cells can be seen in different stages of contact with the significantly larger HEK 293T cells. The extended filament structures at the interface between the cells indicate a possible formation of the NK-cell target-cell immune synapse. Further results, including tomography of the interaction between a HEK 293T cell and two NK-cells, are provided in Paper B.

\subsection{Giant DNA virus infection}

The possibility to image viruses inside a host cell, without sectioning or in other ways altering the cell, is a powerful tool in studies of viral infections. Traditionally, viruses have been viewed as infectious agents without metabolism that can pass through sterilizing filters, and that are too small to be seen in a visible-light microscope [131]. This view has been challenged in the $21^{\text {st }}$ century by the discovery of giant DNA viruses, which have dimensions up to $1.5 \mathrm{\mu m}$ and a genome as complex as many cellular microbes 132 133.

The Stockholm laboratory x-ray microscope was used to study a newly discovered giant DNA virus, Cedratvirus lurbo, belonging to the Pithoviridae family (Paper D). The viral infection dynamics was studied by infecting Acanthamoeba castellanii and imaging a large number of cells at infection times ranging from 6 to 72 hours. In addition, uninfected cells and isolated viral particles were imaged and used as reference. Figure 5.4 shows three examples of cryofixed Acanthamoeba castellanii at different stages of infection. The cell in Fig. 5.4(a) shows no signs of infection, and an overall 

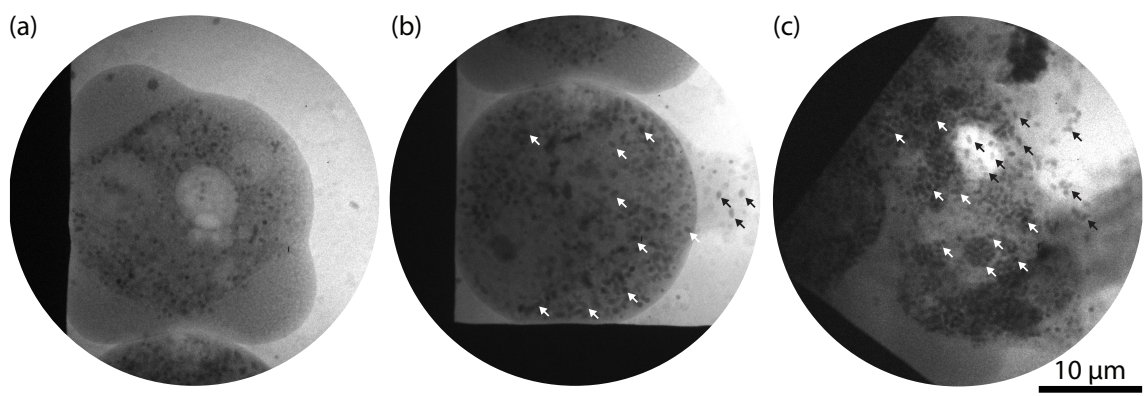

Figure 5.4. Laboratory cryo x-ray microscopy of giant DNA virus infected Acanthamoeba Castellanii. (a) $30 \mathrm{~s}$ exposure of a cell with no visible signs of infection. (b) $60 \mathrm{~s}$ exposure of infected cell, where the ovoid viral particles can be seen throughout the cytoplasm. (c) $60 \mathrm{~s}$ exposure of a heavily infected cell, where the viral particles take up a large part of the cell volume, and the cell shows signs of lysis. In (b) and (c) some viral particles are marked by black and white arrows. Scale bar $10 \mu \mathrm{m}$.

healthy appearance. The cells in Fig. 5.4(b)-(c) contain numerous viral particles, $\sim 0.5 \times 1.5 \mathrm{\mu m}^{2}$ in size, distributed throughout the cytoplasm. Some of the viral particles are marked by arrows. Further results and discussions, including calculations of the bioconversion from cellular host to virus, are presented in Paper D.

To date, very few other studies of viral replication in whole cells have been conducted using soft x-ray microscopy [134], and (to the Author's knowledge) none using laboratory-based x-ray microscopy. The great potential of this method, compared to the more commonly used TEM, is not only due to the fact that the cells are kept unsectioned, unstained, and hydrated. Soft x-ray microscopy also enables imaging of large cohorts of cells, within reasonable experiment times, which is needed in order to gather statistical support for biological conclusions. The high contrast together with a large number of imaged samples allow for comprehensive quantitative studies of viral replication.

\subsection{Glycocalyx of THP-1 cells}

In an ongoing collaboration with the Berlin Laboratory for innovative X-ray Technologies (BliX) at Technische Universität (TU) Berlin, the glycocalyx (GCX) of THP-1 cells is being studied. The GCX is a membrane structure on the surface of the cell, consisting mainly of proteoglycans and hyaluronic acid 135. It has been shown to be extremely fragile and is therefore destroyed by chemical fixation techniques, commonly used in TEM 135 136]. The ability to visualize the GCX of cells in a cryofixed near-native state, is 
therefore a potential advantage of water-window x-ray microscopy.

The THP-1 cells are mainly used as model system. In a later stage, this study aims at investigating the GCX of endothelial cells [136]. These are barrier cells, found in blood vessels and lymphatic vessels, which rely on the GCX to support their barrier function. Studying the GCX of endothelial cells is important for the investigation of inflammatory diseases such as atherosclerosis.

First imaging of cryofixed THP-1 cells has been done using the Stockholm laboratory x-ray microscope and a similar microscope system in Berlin. Results are presented in Paper F. Further imaging, with improved sample preparation to better preserve the GCX, is currently in the planning stage.

\subsection{Future applications}

Due to the great potential of the Stockholm laboratory x-ray microscope, the planned applications are many. In a collaborative project within the Biomedical and X-Ray Physics group at KTH, the cytotoxicity of newly developed nanoparticles is being studied. Some nanoparticles have already been used for x-ray fluorescence computed tomography (XFCT) in whole mice, where they provide functional information by accumulating in certain organs and tumors [137. This particular study used $\mathrm{MoO}_{2}$ particles of $\sim 6 \mathrm{~nm}$ in diameter, which typically agglomerated in $\sim 50 \mathrm{~nm}$ clusters. In a proof-of-principle study of live-mice XFCT, the toxicity of the nanoparticles was deemed negligible for the used concentrations [138. Nevertheless, further analysis at a cellular level is necessary. The Stockholm laboratory x-ray microscope is well suited for imaging of metal nanoparticles in whole cells. The high attenuation in metals (e.g. molybdenum) will provide necessary contrast down to few tens of $\mathrm{nm}$ [28], as long as the resolution is sufficient. Imaging of model cells exposed to a varying concentration of nanoparticles will reveal whether the particles enter into the cells, if they dissolve, or if they accumulate in certain parts. The study can be extended to include several types of nanoparticles, which are currently being developed for XFCT 139.

Another future project is to study virus-infected HEK 293 cells, following the methods developed in Paper D. HEK 293 cells are currently used in the biotechnology industry, e.g., to produce virus for gene therapy. Optimization of the virus production, in order to decrease the overall production cost, includes making sure that the host cells survive for as long as possible. In this project, virus-infected HEK 293 cells will be studied to better understand the mechanisms behind their death. Even though the virus, in this case, is too small to be resolved by soft x-ray microscopy, the obtained cell-morphological information can hopefully provide the needed insight. 


\section{Chapter 6}

\section{Conclusions and outlook}

This Thesis presents the first comprehensive biological studies using laboratory water-window microscopy. The results were enabled by extensive work on upgrading the Stockholm laboratory x-ray microscope with regard to $\mathrm{x}$-ray flux and operational stability, as well as development of new methods for sample preparation.

The stability of the laser-plasma x-ray source has been improved by introducing a radiative heating element to counteract disturbances due to non-uniform freezing of the liquid-nitrogen jet. New routines to avoid disruptive clogs, as well as closer monitoring of the laser focus and related optics have also resulted in a more reliable source operation. Nevertheless, more can be done to improve the source stability further. Current projects include improving the quality and pointing stability of the laser beam, as well as upgrading the laser entrance window arrangement.

A new multilayer condenser mirror, in combination with the improved source stability, has increased the photon flux and resulted in shorter exposure times. This has enabled the first routine 10-second exposure imaging of whole cryofixed cells, as well as the first whole-cell cryotomography with 20 minutes total exposure time. Future work will be done on automating the tomographic imaging procedure to remove overhead time, arising from manual repositioning and refocusing during sample rotation. The increased flux from the source also allows microscope operation at lower powers, which puts less strain on the microscope and improves the stability over time. This has proven especially useful for extensive biological studies where a large number of samples are studied over weeks, or even months.

Finally, work has been done on investigating and improving the mechanical and thermal stability of the Stockholm laboratory x-ray microscope. This not only resulted in an improved image resolution, but also newly developed routines for handling cryogenic samples during microscope 
operation.

The future biological applications are both exciting and promising. The Stockholm laboratory x-ray microscope is well suited for imaging metal nanoparticles in whole cells, with a high natural contrast of particles down to few tens of nm. Here, the newly achieved resolution down to $25 \mathrm{~nm}$ half period will be especially useful. Previous results have also shown the microscope's ability to image cell-morphological changes during virus infections. These results, together with experiences in preparing infected cell samples, will be advantageous for future studies. Furthermore, the recently improved microscope reliability will allow studies of a large number of samples, which will be necessary in order to vary sample parameters, regardless of the application. 


\section{Summary of papers}

This Thesis is based on the six papers listed below. The results include development and improvements of the Stockholm laboratory x-ray microscope, as well as development of new methods for biological soft x-ray microscopy and subsequent analysis. The author was the main responsible for Papers C, D and E. In Papers A and B the author contributed to the design and execution of the experiments, analysis of theoretical and experimental results, and writing of the manuscript. The sample preparation and confocal microscopy in Paper B was mainly done by others. In paper F, the author contributed to the imaging experiments performed at the Stockholm laboratory x-ray microscope and writing of the manuscript.

\section{Paper A: Stability of liquid-nitrogen-jet laser-plasma targets}

In this paper, the stability of the liquid-nitrogen-jet x-ray source is evaluated and improved. Non-uniform freezing of the jet, due to evaporative cooling in combination with imperfections in the glass nozzles, are identified as the cause of the frequently occurring instabilities. Two solutions are presented: increasing the ambient pressure to about $100 \mathrm{mbar}$, and introducing a resistive heating wire to counteract initial freezings at the nozzle orifice. The latter is more suitable, due to the attenuation of soft x-rays in nitrogen gas.

\section{Paper B: Laboratory cryo $x$-ray microscopy for 3D cell imaging}

This paper presents two biological imaging applications using the Stockholm laboratory x-ray microscope, made possible by improvements of the x-ray source stability and new condenser optics. HEK 293T cells were imaged under different stages of starvation and the interaction between NK cells and HEK 293T cells was imaged. Three tomographic datasets were acquired and reconstructed, consisting mainly of 10-second exposures. 


\section{Paper C: Stability investigation of a cryo soft $x$-ray microscope by fiber interferometry}

This paper presents stability measurements of the most sensitive imaging components in the Stockholm laboratory x-ray microscope, using fiberbased laser interferometry. High-frequency vibrations, originating mainly from a turbo pump, are shown to limit the achievable imaging resolution. In addition, thermal drifts of the cryo sample holder are shown to affect the resolution, if not adequately handled. Vibration damping modifications to the setup are shown to improve the resolution from $35-40 \mathrm{~nm}$ to about 25 nm half period.

\section{Paper D: Quantitative bioconversion in giant DNA virus infection}

This paper presents a quantitative study of the replication of a previously unknown giant DNA virus, named Cedratvirus lurbo, inside an amoebal host. A large number of laboratory x-ray microscopy images are presented and analyzed to calculate the burst size, i.e., the number of viral particles produced per cell, and the average conversion of biomass from host to virus. Classical end-point dilution titrations together with thermogravimetric analysis is used to verify the results.

\section{Paper E: Laboratory water-window x-ray microscopy}

This is mainly a review paper, but with some additional new material. It contains evaluations and comparisons of all relevant parts of a laboratory soft x-ray microscope, including source, optics, sample and detector, as well as a complete system consideration. Two laboratory x-ray microscopes, located at KTH Stockholm and BliX/TU Berlin, are presented in more detail. Finally, a range of new and old applications, from these two microscopes, are summarized.

\section{Paper F: Laboratory soft $\mathrm{x}$-ray microscopy with an integrated visible- light microscope - Correlative workflow for faster 3D cell imaging}

This paper presents the integration of a visible-light microscope into the setup of the laboratory x-ray microscope at BliX/TU Berlin. The visiblelight microscope has two purposes: To provide an overview of the sample and to enable alignment of the sample rotation axis with the optical axis of the x-ray microscope, which gives a faster workflow for tomographic imaging. The advantages of the improved setup are demonstrated by tomographic (3D) imaging of a diatom and (2D) imaging of cryofixed THP-1 cells. The latter was done both in Berlin and in Stockholm. 


\section{Acknowledgements}

There are many, without whom this work would not have been possible, and who therefore deserve special recognition. I wish to express my sincerest gratitude to my supervisor Hans Hertz. Thank you for your hard work and encouraging attitude. Communication with you has always been easy and straightforward, which is something that I much appreciate. The same goes for my co-supervisors Ulrich Vogt and Jonas Sellberg. Thank you for everything from detailed theoretical discussions to practical efforts in the laboratory.

I would like to thank my closest colleagues Emelie Fogelqvist and Mårten Selin, who made me feel welcome from day one. Thank you Mårten for discussions on optics and for providing excellent Labview and Matlab support. Thank you Emelie for your hard work, creative ideas, and positive attitude during long hours in the laboratory. Many thanks to my newest colleague Komang Arsana, who will take over responsibility of the microscope and its applications. I am confident that your focused work and genuine interest will lead to many interesting results. To our past and present team members Jussi Rahomäki, Anders Holmberg, Thomas Frisk, Martin Svenda and Björn Hessmo, thank you for productive discussions during our Friday meetings. You have all contributed to this work in various ways.

Modern research is highly dependent on collaborations across groups and across borders. Many thanks to our collaborators in Berlin, Aurélie Dehlinger, Christian Seim, and Holger Stiel. Thank you for fun and rewarding discussions and for a pleasant visit in Berlin. Furthermore, I would like to acknowledge the contributions from my co-authors Björn Önfelt, Valentina Carannante, Hemanth Reddy, Bejan Hamawandi, and Muhammet Toprak.

Finally, my deepest gratitude to my friends and family who supported me during these years. To my parents, for your love, good advice and for never questioning any of my decisions. To Kristin, my dear wife, who always listens and helps sort out my thoughts, even now when two small children take up much of our time. To Signe and Tage, who I hope will one day read this. I love you. 



\section{References}

[1] A. J. M. Wollman, R. Nudd, E. G. Hedlund, and M. C. Leake, From Animaculum to single molecules: 300 years of the light microscope, Open Biol. 5, 150019 (2015).

[2] N. Lane, The unseen World: Reflections on Leeuwenhoek (1677) 'Concerning little animals', Philos. Trans. R. Soc. B 370, 20140344 (2015).

[3] R. Hooke, Micrographia, Project GutenBerg, http://www.gutenberg.org (2005).

[4] E. Abbe, Beiträge zur Theorie des Mikroskops und der mikroskopischen Wahrnehmung, Arch. Mikrosk. Anat. 9, 413-468 (1873).

[5] A. Köhler, Ein neues Beleuchtungsverfahren für mikrophotographische Zwecke, Zeitschrift für wissenschaftliche Mikroskopie und für Mikroskopische Technik 10, 433-440 (1893).

[6] E. Hecht, Optics, Pearson, Global Edition, 5th edition (2017).

[7] L. Schermelleh, A. Ferrand, T. Huser, C. Eggeling, M. Sauer, O. Biehlmaier, and G. P. Drummen, Super-resolution microscopy demystified, Nat. Cell Biol. 21, 72-84 (2019).

[8] C. Jacobsen, X-ray Microscopy, Cambridge University Press (2019).

[9] L. de Broglie, The reinterpretation of wave mechanics, Found. Phys. 1, 5-15 (1970).

[10] R. Erni, M. D. Rossell, C. Kisielowski, and U. Dahmen, Atomicresolution imaging with a sub-50-pm electron probe, Phys. Rev. Lett. 102, 096101 (2009).

[11] X. Li et al., Electron counting and beam-induced motion correction enable near-atomic-resolution single-particle cryo-EM, Nat. Methods 10, 584-590 (2013). 
[12] D. C. Joy and J. B. Pawly, High-resolution scanning electron microscopy, Ultramicroscopy 47, 80-100 (1992).

[13] K. Miranda, W. Girard-Dias, M. Attias, W. de Souza, and I. Ramos, Three dimensional reconstruction by electron microscopy in the life sciences: An introduction for cell and tissue biologists, Mol. Reprod. Dev. 82, 530-547 (2015).

[14] N. Jalili and K. Laxminarayana, A review of atomic force microscopy imaging systems: Application to molecular metrology and biological sciences, Mechatronics 14, 907-945 (2004).

[15] M. Radmacher, Measuring the elastic properties of biological samples with the AFM, IEEE Eng. Med. Biol. Mag. 16, 47-57 (1997).

[16] D. Baddeley and J. Bewersdorf, Biological Insight from SuperResolution Microscopy: What We Can Learn from Localization-Based Images, Annu. Rev. Biochem. 87, 965-989 (2018).

[17] S. W. Hell and J. Wichmann, Breaking the diffraction resolution limit by stimulated emission: stimulated-emission-depletion fluorescence microscopy, Opt. Lett. 19, 780-782 (1994).

[18] E. Betzig et al., Imaging intracellular fluorescent proteins at nanometer resolution, Science 313, 1642-1645 (2006).

[19] M. Bates, B. Huang, G. T. Dempsey, and X. Zhuang, Multicolor superresolution imaging with photo-switchable fluorescent probes, Science 317, 1749-1753 (2007).

[20] F. Göttfert, C. A. Wurm, V. Mueller, S. Berning, V. C. Cordes, A. Honigmann, and S. W. Hell, Coaligned dual-channel STED nanoscopy and molecular diffusion analysis at $20 \mathrm{~nm}$ resolution, Biophys. J. 105, L01-L03 (2013).

[21] M. Sauer and M. Heilemann, Single-Molecule Localization Microscopy in Eukaryotes, Chem. Rev. 117, 7478-7509 (2017).

[22] G. Vicidomini, P. Bianchini, and A. Diaspro, STED super-resolved microscopy, Nat. Methods 15, 173-182 (2018).

[23] C. Eggeling, K. I. Willig, S. J. Sahl, and S. W. Hell, Lens-based fluorescence nanoscopy, Q. Rev. Biophys. 48, 178-243 (2015).

[24] W. C. Röntgen, Über eine neue Art von Strahlen (Vorläufige Mittheilung), Sitzungsber. Physik. Med. Ges. Würzburg, 132-141 (1895). 
[25] A. Feldman, A sketch of the technical history of radiology from 1896 to 1920., Radiographics 9, 1113-1128 (1989).

[26] P. P. Ewald, Fifty years of $x$-ray diffraction, International Union of Crystallography, Reidel, Dordrecht. (1962).

[27] D. T. Attwood and A. Sakdinawat, X-Rays and Extreme Ultraviolet Radiation: Principles and Applications, chapter 3, pages 60-109, Cambridge University Press, 2nd edition (2016).

[28] B. L. Henke, E. M. Gullikson, and J. C. Davis, X-ray interactions: photoabsorption, scattering, transmission, and reflection at $E=50$ 30000 eV, Z=1-92, At. Data Nucl. Data Tables 54, 181 (1993). Available at http://henke.lbl.gov/optical_constants/.

[29] E. Spiller, R. Feder, D. Topalian, D. Eastman, W. Gudat, and

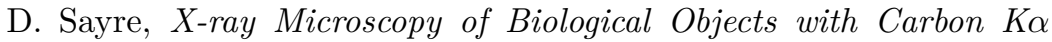
and with Synchrotron Radiation, Science 191, 1172-1174 (1976).

[30] B. Niemann, D. Rudolph, and G. Schmahl, for Microscopic and Spectroscopic Applications, Opt. Commun. 12, 160-163 (1974).

[31] P. Horowitz and J. A. Howell, A Scanning X-Ray Microscope Using Synchrotron Radiation, Science 178, 608-611 (1972).

[32] A. Sakdinawat and D. Attwood, Nanoscale X-ray imaging, Nat. Photonics 4, 840-848 (2010).

[33] V. Weinhardt, J. H. Chen, A. Ekman, G. McDermott, M. A. Le Gros, and C. Larabell, Imaging cell morphology and physiology using X-rays, Biochem. Soc. Trans. 47, 324-332 (2019).

[34] W. Chao, B. D. Harteneck, J. A. Liddle, E. H. Anderson, and D. T. Attwood, Soft X-ray microscopy at a spatial resolution better than 15 nm, Nature 435, 1210-1213 (2005).

[35] B. Rösner et al., 7 nm Spatial Resolution in Soft X-ray Microscopy, Microsc. Microanal. 24, 270-271 (2018).

[36] H. N. Chapman and K. A. Nugent, Coherent lensless X-ray imaging, Nat. Photonics 4, 833-839 (2010).

[37] J. Miao, P. Charalambous, J. Kirz, and D. Sayre, Extending the methodology of X-ray crystallography to non-crystalline specimens, Nature 400, 342-344 (2003). 
[38] I. Mcnulty, J. Kirz, C. Jacobsen, E. H. Anderson, M. R. Howells, and D. P. Kern, High-Resolution Imaging by Fourier Transform X-ray Holography, Science 256, 1009-1012 (1992).

[39] H. N. Chapman, Phase-retrieval X-ray microscopy by Wignerdistribution deconvolution, Ultramicroscopy 66, 153-172 (1996).

[40] D. T. Attwood and A. Sakdinawat, X-Rays and Extreme Ultraviolet Radiation: Principles and Applications, chapter 2, pages 27-59, Cambridge University Press, 2nd edition (2016).

[41] A. C. Thompson et al., X-ray Data Booklet, Lawrence Berkeley National Laboratory, 3rd edition (2009). Available at http://xdb.lbl.gov/.

[42] M. Bergh, G. Huldt, N. Tîmneanu, F. R. Maia, and J. Hajdu, Feasibility of imaging living cells at subnanometer resolutions by ultrafast $X$-ray diffraction, Q. Rev. Biophys. 41, 181-204 (2008).

[43] J. W. Goodman, Fourier optics, Springer, Berlin-Heidelberg, 3rd edition (2008).

[44] W. C. Nixon, High-resolution X-ray projection microscopy, Proc. R. Soc. Lond. A 232, 475-485 (1955).

[45] K. Yada and S. Takahashi, Target materials suitable for projection $X$-ray microscope observation of biological samples, J. Elect. Micros. 38, 321-331 (1989).

[46] G. Kirchhoff, Zur Theorie der Lichtstrahlen, Ann. Phys. 18, 663-695 (1882).

[47] A. Sommerfeld, Mathematische Theorie der Diffraktion, Math. Ann. 47, 317-347 (1886).

[48] M. Born and E. Wolf, Principles of Optics, chapter 10, pages 554-632, Cambridge University Press, 7th edition (1999).

[49] D. T. Attwood and A. Sakdinawat, X-Rays and Extreme Ultraviolet Radiation: Principles and Applications, chapter 4, pages 110-147, Cambridge University Press, 2nd edition (2016).

[50] D. T. Attwood and A. Sakdinawat, X-Rays and Extreme Ultraviolet Radiation: Principles and Applications, chapter 10, pages 446-513, Cambridge University Press, 2nd edition (2016). 
[51] J. Miao, T. Ishikawa, I. K. Robinson, and M. M. Murnane, Beyond crystallography: Diffractive imaging using coherent $X$-ray light sources, Science 348, 530-535 (2015).

[52] T. M. Buzug, Computed tomography, Springer, Berlin-Heidelberg (2008).

[53] J. T. Bushberg, A. J. Seibert, J. E. M. Leidholdt, and J. M. Boone, The essential physics of medical imaging, Open Biol. (2012).

[54] J. Radon, Über die bestimmung von funktionen durch ihre integralwerte langs geweisser mannigfaltigkeiten (on the determination of functions from their integrals along certain manifolds), Ber. Verh. Sachs. Akad. Wiss. Leipzig, Math.-Phys. Kl. 69, 262-277 (1917).

[55] R. Gordon, R. Bender, and G. T. Herman, Algebraic Reconstruction Techniques (ART) for three-dimensional electron microscopy and $X$ ray photography, J. Theor. Biol. 29, 471-481 (1970).

[56] A. C. Kak and M. Slaney, Principles of Computerized Tomographic Imaging, Society of Industrial and Applied Mathematics (2001).

[57] R. A. Crowther, D. J. DeRosier, and F. R. S. Klug, The reconstruction of a three-dimensional structure from projections and its application to electron microscopy. II. Direct methods., Proc. R. Soc. B 182, 89-102 (1972).

[58] P. Penczek, M. Marko, K. Buttle, and J. Frank, Double-tilt electron tomography, Ultramicroscopy 60, 393-410 (1995).

[59] K. J. Batenburg and J. Sijbers, DART: a practical reconstruction algorithm for discrete tomography., IEEE Trans. Image Process 20, 2542-2553 (2011).

[60] Y. Deng, Y. Chen, Y. Zhang, S. Wang, F. Zhang, and F. Sun, ICON: $3 D$ reconstruction with 'missing-information' restoration in biological electron tomography, J. Struct. Biol. 195, 100-112 (2016).

[61] M. Selin, E. Fogelqvist, S. Werner, and H. M. Hertz, Tomographic reconstruction in soft $x$-ray microscopy using focus-stack backprojection, Opt. Lett. 40, 2201 (2015).

[62] D. T. Attwood, K. Halbach, and K. Kwang-Je, Tunable Coherent Xrays, Science 228, 1265-1272 (1985). 
[63] D. Attwood and A. Sakdinawat, X-Rays and Extreme Ultraviolet Radiation: Principles and Applications, chapter 5, pages 148-226, Cambridge University Press, 2nd edition (2016).

[64] D. T. Attwood and A. Sakdinawat, X-Rays and Extreme Ultraviolet Radiation: Principles and Applications, chapter 6, pages 227-278, Cambridge University Press, 2nd edition (2016).

[65] B. W. Mcneil and N. R. Thompson, X-ray free-electron lasers, Nat. Photonics 4, 814-821 (2010).

[66] G. Geloni, Z. Huang, and C. Pellegrini, The Physics and Status of $X$-ray Free-electron Lasers, in U. Bergmann, V. K. Yachandra, and J. Yano (editors), X-Ray Free-Electron Lasers, pages 1-44, The Royal Society of Chemistry (2017).

[67] H. N. Chapman et al., Femtosecond X-ray protein nanocrystallography, Nature 470, 73-78 (2011).

[68] G. Schmahl et al., A Laboratory X-Ray Microscope with a Plasma $X$-Ray Source, in A. G. Michette, G. R. Morrison, and C. J. Buckley (editors), X-Ray Microscopy III, pages 66-69, Springer, BerlinHeidelberg (1992).

[69] W. Neff, J. Eberle, R. Holz, F. Richter, and R. Lebert, A Plasma Focus as Radiation Source for a Laboratory X-Ray Microscope, in D. Sayre, J. Kirz, M. Howells, and H. Rarback (editors), X-Ray Microscopy II, volume 56, pages 22-29, Springer, Berlin-Heidelberg (1988).

[70] R. Lebert, W. Neff, and D. Rothweiler, Pinch plasma source for X-ray microscopy with nanosecond exposure time, J. X-Ray Sci. Technol. 6, 107-140 (1996).

[71] D. T. Attwood and A. Sakdinawat, X-Rays and Extreme Ultraviolet Radiation: Principles and Applications, chapter 8, pages 315-402, Cambridge University Press, 2nd edition (2016).

[72] M. Benk, K. Bergmann, D. Schäfer, and T. Wilhein, Compact soft $x$-ray microscope using a gas-discharge light source, Opt. Lett. 33, 2359 (2008).

[73] D. B. Carlson, J. Gelb, V. Palshin, and J. E. Evans, Laboratory-based cryogenic soft $x$-ray tomography with correlative cryo-light and electron microscopy, Microsc. Microanal. 19, 22-29 (2013). 
[74] R. Kodama, K. Okada, N. Ikeda, M. Mineo, K. A. Tanaka, T. Mochizuki, and C. Yamanaka, Soft x-ray emission from $\omega 0,2 \omega 0$, and $4 \omega 0$ laser-produced plasmas, J. Appl.Phys. 59, 3050-3052 (1986).

[75] M. Kishimoto, M. Kado, M. Ishino, S. Tamotsu, K. Yasuda, and K. Shinohara, Development of single shot soft $x$-ray contact microscopy system for nano-scale dynamics measurement of living biological specimen, AIP Conf. Proc. 1465, 43-47 (2012).

[76] T. Ogata, K. Iimura, S. Aoki, Y. Yoshidomi, O. Mitomi, and K. Shinada, Development of a Grazing Incidence Soft X-ray Microscope with a Laser-Produced Plasma Source, J. Elec. Spectr. Rel. Phenom. 80, 357-360 (1996).

[77] M. Hoshino and S. Aoki, Laser plasma soft x-ray microscope with wolter mirrors for observation of biological specimens in air, Jpn. J. Appl. Phys. 45, 989-994 (2006).

[78] A. G. Michette et al., Scanning x-ray microscopy using a laser-plasma source, Rev. Sci. Instrum. 64, 1478-1482 (1993).

[79] L. Rymell, M. Berglund, and H. M. Hertz, Debris-free single-line laser-plasma x-ray source for microscopy, Appl. Phys. Lett. 66, 2625-2627 (1995).

[80] M. Berglund, L. Rymell, M. Peuker, T. Wilhein, and H. M. Hertz, Compact water-window transmission X-ray microscopy, J. Microsc. 197, 268-273 (2000).

[81] H. Fiedorowicz, A. Bartnik, Z. Patron, and P. Parys, X-ray emission from laser-irradiated gas puff targets, Appl. Phys. Lett. 62, 2778-2780 (1993).

[82] P. Wachulak et al., A Compact "Water Window" Microscope with 60 nm Spatial Resolution for Applications in Biology and Nanotechnology, Microsc. Microanal. 21, 1214-1223 (2015).

[83] D. H. Martz et al., High average brightness water window source for short-exposure cryomicroscopy., Opt. Lett. 37, 4425-4427 (2012).

[84] M. Berglund, L. Rymell, H. M. Hertz, and T. Wilhein, Cryogenic liquid-jet target for debris-free laser-plasma soft x-ray generation, Rev. Sci. Instrum. 69, 2361-2364 (1998).

[85] J. F. Adam, J. P. Moy, and J. Susini, Table-top water window transmission x-ray microscopy: Review of the key issues, and conceptual 
design of an instrument for biology, Rev. Sci. Instrum. 76, 091301 (2005).

[86] M. O. Krause, Atomic radiative and radiationless yields for $K$ and $L$ shells, J. Phys. Chem. Ref. Data 8, 307-327 (1979).

[87] P. Cheng, R. Feder, D. Shinozaki, K. Tan, R. Eason, A. Michette, and R. Rosser, Soft X-ray Contact Microscopy, Nucl. Instrum. Methods Phys. Res. A 246, 668-674 (1986).

[88] K. Murakami, T. Oshino, H. Nakamura, M. Ohtani, and H. Nagata, Related content Normal-Incidence X-Ray Microscope for Car-

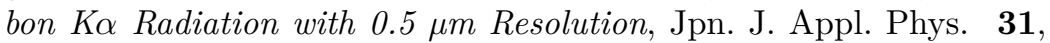
L1500-L1502 (1992).

[89] S. Ohsuka et al., Laboratory-size three-dimensional x-ray microscope with Wolter type $i$ mirror optics and an electron-impact water window x-ray source, Rev. Sci. Instrum. 85, 093701 (2014).

[90] P. Skoglund, U. Lundström, U. Vogt, and H. M. Hertz, Highbrightness water-window electron-impact liquid-jet microfocus source, Appl. Phys. Lett. 96, 084103 (2010).

[91] V. Cardin et al., Self-channelled high harmonic generation of water window soft x-rays, Journal of Physics B 51, 174004 (2018).

[92] G. K. Tadesse et al., High speed and high resolution table-top nanoscale imaging, Optics Letters 41, 5170 (2016).

[93] A. Rockwood, Y. Wang, S. Wang, M. Berrill, V. N. Shlyaptsev, and J. J. Rocca, Compact gain-saturated $x$-ray lasers down to $685 \mathrm{~nm}$ and amplification down to $585 \mathrm{~nm}$, Optica 5, 257 (2018).

[94] M. Zürch et al., Transverse Coherence Limited Coherent Diffraction Imaging using a Molybdenum Soft X-ray Laser Pumped at Moderate Pump Energies, Scientific Reports 7, 1-10 (2017).

[95] F. Albert and A. G. Thomas, Applications of laser wakefield accelerator-based light sources, Plasma Physics and Controlled Fusion 58 (2016).

[96] F. Brizuela et al., Microscopy of extreme ultraviolet lithography masks with 132 nm tabletop laser illumination, Optics Letters 34, 271-273 (2009). 
[97] T. Helk, M. Zürch, and C. Spielmann, Perspective: Towards single shot time-resolved microscopy using short wavelength table-top light sources, Structural Dynamics 6, 010902 (2019).

[98] C. Burcklen, S. de Rossi, E. Meltchakov, D. Dennetière, B. Capitanio, F. Polack, and F. Delmotte, High-reflectance magnetron-sputtered scandium-based $x$-ray multilayer mirrors for the water window, Opt. Lett. 42, 1927-1930 (2017).

[99] E. M. Gullikson, F. Salmassi, A. L. Aquila, and F. Dollar, Progress in short period multilayer coatings for water window applications, in International conference on the physics of $x$-ray multilayer structures (2006).

[100] T. Feigl et al., Multilayer Collector Optics for Water Window Microscopy, Presentation at the International Workshop on EUV and soft X-Ray Sources, (2015, November).

[101] P. Kirkpatrick and A. V. Baez, Formation of Optical Images by XRays, J. Opt. Soc. Am. 38, 766-774 (1948).

[102] X. Zeng, F. Duewer, M. Feser, C. Huang, A. Lyon, A. Tkachuk, and W. Yun, Ellipsoidal and parabolic glass capillaries as condensers for x-ray microscopes, Appl. Opt. 47, 2376-2381 (2008).

[103] H. Wolter, Spiegelsysteme streifenden Einfalls als abbildende Optiken für Röntgenstrahlen, Ann. Phys. 445, 94-114 (1952).

[104] P. Guttmann, X. Zeng, M. Feser, S. Heim, W. Yun, and G. Schneider, Ellipsoidal capillary as condenser for the BESSY full-field X-ray microscope, J. Phys. Conf. Ser. 186, 012064 (2009).

[105] A. Sorrentino et al., MISTRAL: A transmission soft X-ray microscopy beamline for cryo nano-tomography of biological samples and magnetic domains imaging, J. Synchrotron Radiat. 22, 1112-1117 (2015).

[106] K. W. Kim et al., Compact soft x-ray transmission microscopy with sub-50 nm spatial resolution, Phys. Med. Biol. 51, N99-N107 (2006).

[107] E. H. Anderson and D. Kern, Nanofabrication of Zone Plates for X-Ray Microscopy, in A. G. Michette, G. R. Morrison, and C. J. Buckley (editors), X-Ray Microscopy III, pages 75-78, Springer Berlin Heidelberg (1992).

[108] W. Yun and others., Nanometer focusing of hard $x$ rays by phase zone plates, Rev. Sci. Instrum. 70, 2238-2241 (1999). 
[109] J. M. Heck, D. T. Attwood, W. Meyer-Ilse, and E. H. Anderson, Resolution determination in X-ray microscopy: An analysis of the effects of partial coherence and illumination spectrum, J. X-ray Sci. Technol. 8, 95-104 (1998).

[110] M. A. Le Gros et al., Biological soft X-ray tomography on beamline 2.1 at the Advanced Light Source, J. Synchrotron Radiat. 21, 1370-1377 (2014).

[111] H. Legall et al., Compact x-ray microscope for the water window based on a high brightness laser plasma source, Opt. Express 20, 18362-18369 (2012).

[112] D. Weiß, G. Schneider, B. Niemann, P. Guttmann, D. Rudolph, and G. Schmahl, Computed tomography of cryogenic biological specimens based on X-ray microscopic images, Ultramicroscopy 84, 185-197 (2000).

[113] M. A. Le Gros, G. McDermott, and C. A. Larabell, X-ray tomography of whole cells, Curr. Opin. Struct. Biol. 15, 593-600 (2005).

[114] J. Maser et al., Soft X-ray microscopy with a cryo scanning transmission $X$-ray microscope: I. Instrumentation, imaging and spectroscopy, J. Microsc. 197, 68-79 (2000).

[115] G. Schneider, Cryo X-ray microscopy with high spatial resolution in amplitude and phase contrast, Ultramicroscopy 75, 85-104 (1998).

[116] G. Schneider, B. Niemann, P. Guttmann, D. Rudolph, and G. Schmahl, Cryo X-ray Microscopy, Synchrotron Radiat. News 8, 19-28 (1995).

[117] K. P. Ryan, D. H. Purse, S. G. Robinson, and J. W. Wood, The relative efficiency of cryogens used for plunge-cooling biological specimens, J. Microsc. 145, 89-96 (1987).

[118] J. Kirz, C. Jacobsen, and M. Howells, Soft X-ray microscopes and their biological applications, Q. Rev. of Biophys. 28, 33-130 (1995).

[119] S. Jearanaikoon and J. V. Abraham-Peskir, An X-ray microscopy perspective on the effect of glutaraldehyde fixation on cells, J. Microsc. 218, 185-192 (2005).

[120] S. Williams, X. Zhang, C. Jacobsen, J. Kirz, S. Lindaas, J. Van't Hof, and S. S. Lamm, Measurements of wet metaphase chromosomes in the scanning transmission X-ray microscope, J. Microsc. 170, 155-165 (1993). 
[121] H. M. Hertz, M. Bertilson, O. von Hofsten, S. C. Gleber, J. Sedlmair, and J. Thieme, Laboratory X-ray microscopy for high-resolution imaging of environmental colloid structure, Chem. Geol. 329, 26-31 (2012).

[122] See, e.g., https://andor.oxinst.com/products/high-energy-detection/ ikon-l-so.

[123] A. L. Kilcoyne et al., Interferometer-controlled scanning transmission $X$-ray microscopes at the Advanced Light Source, J. Synchrotron Radiat. 10, 125-136 (2003).

[124] T. Wilhein, D. Hambach, B. Niemann, M. Berglund, L. Rymell, and H. M. Hertz, Off-axis reflection zone plate for quantitative soft $\{X\}$ ray source characterization, Appl. Phys. Lett. 71, 190-192 (1997).

[125] P. Russbueldt et al., Innoslab Amplifiers, IEEE Journal of Selected Topics in Quantum Electronics 21, 447-463 (2015).

[126] T. Feigl, personal communication, (2016, June), http://www.optixfab.com/.

[127] S. T. Shibutani and T. Yoshimori, A current perspective of autophagosome biogenesis., Cell Res. 24, 58-68 (2014).

[128] Y. Liu and B. Levine, Autosis and autophagic cell death: the dark side of autophagy., Cell Death Differ. 22, 367-76 (2015).

[129] J. S. Orange, Formation and function of the lytic NK-cell immunological synapse., Nat. Rev. Immunol. 8, 713-725 (2008).

[130] E. M. Mace et al., Cell biological steps and checkpoints in accessing NK cell cytotoxicity, Immunol. Cell Biol. 92, 245-255 (2014).

[131] A. Lwoff, The concept of virus, J. Gen. Microbiol. 17, 239-253 (1957).

[132] B. La Scola et al., A giant virus in amoebae, Science 299, 2033 (2003).

[133] C. Abergel, M. Legendre, and J. M. Claverie, The rapidly expanding universe of giant viruses: Mimivirus, Pandoravirus, Pithovirus and Mollivirus, FEMS Microbiol. Rev. 39, 779-796 (2015).

[134] F. J. Chichón et al., Cryo X-ray nano-tomography of vaccinia virus infected cells, J. Struct. Biol. 177, 202-211 (2012). 
72 | REFERENCES

[135] W. C. Poller et al., Initial interaction of citrate-coated iron oxide nanoparticles with the glycocalyx of THP-1 monocytes assessed by real-time magnetic particle spectroscopy and electron microscopy, Sci.

Rep. 10, 3591 (2020).

[136] E. E. Ebong, F. P. MacAluso, D. C. Spray, and J. M. Tarbell, Imaging the endothelial glycocalyx in vitro by rapid freezing/freeze substitution transmission electron microscopy, Arterioscler. Thromb. Vasc. Biol 31, 1908-1915 (2011).

[137] J. C. Larsson, C. Vogt, W. Vågberg, M. S. Toprak, J. Dzieran, M. Arsenian-Henriksson, and H. M. Hertz, High-spatial-resolution $x$ ray fluorescence tomography with spectrally matched nanoparticles, Phys. Med. Biol. 63, 164001 (2018).

[138] K. Shaker et al., Longitudinal In-Vivo X-Ray Fluorescence Computed Tomography with Molybdenum Nanoparticles, IEEE Trans. Med. Imag., submitted manuscript (2020).

[139] Y. Li, K. Shaker, J. C. Larsson, C. Vogt, H. M. Hertz, and M. S. Toprak, A library of potential nanoparticle contrast agents for $X$-ray fluorescence tomography bioimaging, Contrast Media Mol. Imaging 2018, 1-7 (2018). 